Florida International University FIU Digital Commons

\title{
Colombian Jazz dance suite : a synthesis of the music of the colombian andes and modern jazz
}

Alvaro Bermudez

Florida International University

DOI: $10.25148 /$ etd.FI14051117

Follow this and additional works at: https://digitalcommons.fiu.edu/etd

Part of the Music Theory Commons

\section{Recommended Citation}

Bermudez, Alvaro, "Colombian Jazz dance suite : a synthesis of the music of the colombian andes and modern jazz" (2007). FIU Electronic Theses and Dissertations. 1767.

https://digitalcommons.fiu.edu/etd/1767 


\section{FLORIDA INTERNATIONAL UNIVERSITY}

Miami, Florida

COLOMBIAN JAZZ DANCE SUITE:

A SYNTHESIS OF THE MUSIC OF THE COLOMBIAN ANDES AND MODERN

JAZZ

A thesis submitted in partial fulfillment of the

requirements for the degree of

MASTER OF MUSIC

by

Alvaro Bermudez

2007 
To: Dean Juan Antonio Bueno

College of Architecture and the Arts

This thesis written by Alvaro Bermudez, and entitled Colombian Jazz Dance Suite: a Synthesis of the Music of the Colombian Andes and Modern Jazz, having been approved in respect to style and intellectual content, is referred to you for judgment

We have read this thesis and recommend that it be approved.

Mike Orta

Gary Campbell

Sam Lussier, Major Professor

Date of Defense: July $20^{\text {th }}, 2007$

The thesis of Alvaro Bermudez is approved.

Dean Juan Antonio Bueno

College of Architecture and the Arts

Dean George Walker

University Graduate School

Florida International University, 2007 


\section{ABSTRACT OF THE THESIS \\ COLOMBIAN JAZZ DANCE SUITE:}

A SYNTHESIS OF THE MUSIC OF THE COLOMBIAN ANDES AND

MODERN JAZZ

by

Alvaro Bermudez

Florida International University, 2007

Miami, Florida

Professor Sam Lussier, Major Professor

This thesis is an attempt to unite two distinct and dissimilar musical genres, the music of the Colombian Andes and modern jazz. The compositions to be analyzed in this thesis are meant to function as parts of a whole. Thus, they will be linked by thematic and rhythmic material. In their entirety the pieces will form a suite of dances not unlike those of Baroque composers, with titles that denote the name of the particular air being employed by the composer, who is also the author of this thesis. These individual dances are orchestrated for a jazz ensemble consisting of piano, string bass, drums, alto saxophone, and guitar. The rhythmic underpinning of this work is inspired by the folk music of Colombia and the harmonic content will be derived from the jazz idiom. The purpose of this thesis is to demonstrate the possible product of the fusion of musical disciplines that are on the surface in no way related. This thesis will also attempt to show an example of how cultures can meld socio-artistically. 
TABLE OF CONTENTS

CHAPTER

PAGE

I. INTRODUCTION

1

Il. BAMBUCO

6

III. PASILLO

11

IV. DANZA

15

V. VALS

VI. PASILLO FIESTERO

VII. CURRULAO 26 BIBLIOGRAPHY 29 APPENDIX 


\section{Chapter 1}

\section{INTRODUCTION}

In writing these pieces, the author attempted to reconcile two distinct musical styles, Colombian Folkloric music of the Andean region and modern jazz. Immediately, the necessity arises to state that these diverse musical cultures are both already cultural amalgams created by the migration and acculturation of people.

The African Diaspora is present in both disciplines. Early attempts by Colombian music historians to negate the influence of slave music on their treasured musical patrimony have been debunked by previous authors. ${ }^{1}$ The influence of this same African culture in the conception of jazz music is already well documented.

The two musical styles in question also share European descent, which is most of all noticeable in the harmonic language of each. Distinctions between the inherently tertian and cyclical nature of Colombian music, and the harmonic extensions found in jazz can be traced anthropologically to the differences in the music of the Spanish court from the Baroque and early Classical period whose harmonic influence can be felt in Colombian music, and the music of the French Impressionists which was influential in the Creole culture of New Orleans in the latter half of the nineteenth century. ${ }^{2}$

The main factor that denotes the differences in these styles is the influence of indigenous rhythms belonging to the pre-Columbian cultures that existed in this region of

1 John Varney, "Colombian Bambuco: The evolution of a national music style" (Ph.D. thesis, Queensland Conservatorium, Griffith University, 1999) 290.

2 Frank Tirro, Jaz: A history 
the Andes. No such influence exists in jazz. I chose to use these airs as the motor force for these movements since their appearance in jazz has been sparse. Colombian saxophonist Antonio Arnedo, has done what I believe to be the most mainstream recording of this combination.

The name of each movement comes from the specific air that was used for its rhythmic base. The titles are Bambuco, Pasillo, Vals, Currulao, Pasillo Fiestero and Danza. All of these are well known rhythms in Colombian culture. They have had some degree of dissemination in the broader musical sphere but are not as widely known as other Latin American music.

All, except for the Danza, are triple meters, generally notated in $3 / 4$ time. (6/8 was also used in this suite to facilitate the reading of these ambiguous and syncopated rhythms). The Danza, a duple meter, shares impetus with the Habanera, well known in classical repertoire. Although the majority of the meters are triple in nature, the composer attempted to make each distinct and easily discernible.

Each of these rhythms, in their natural folk state, consists of a repetitive pattern of bass notes and chord punctuation. These patterns are unique to each air. There is a myriad of songs composed in each of these airs. An experienced Colombian musician, having never heard a song in question, only needs to be told which air the song employs and in which key it will be played. The musician will then harmonize the melody he hears using the chords available in that key, while employing the rhythmic signature of the given air. The harmony used in most early Colombian composition is mainly tonal and follows strictly the general rules of cyclical harmony and voice leading. This allows the musician to play a great number of songs though he may only know a few chords. 
This characteristic shows a similarity to early Blues where an enormous amount of music has been written using the same harmonic progression. In fact, the Blues and Colombian music share a rural influence and provenance. They each have a harmonic simplicity they share and are representative of the cultures from which they come. The personalities of each culture are represented in the music.

In preparing these pieces, the author paid a great deal of attention to their melodic detail. As will be discussed in each subsequent chapter, the melodies contain fragments typical of Colombian music. The melody for Danza bears the least resemblance to its counterparts. Being the only inherently duple meter air in the suite, it was chosen by the composer to be the most abstract of the six themes. It is set apart from the others in its melodic contour and in its rhythmic usage. The rest contain rhythmic signatures from the music of Colombia. The author attempted to do this in a way that was not gratuitous and that did not compromise the integrity of the melodies. As Varney charts, there are rhythmic fragments and resolutions that are pervasive in traditional Colombian composition $^{3}$. The author tried to use these fragments sparsely but at key moments during which the Colombian listener would expect them to be there. The author has done extensive performing of and listening to Colombian music. His background is Colombian. He was born in New York, but spent his later teens in the Valle state of southwestern Colombia between the Eastern and Central Ridges of the Andes Mountains. Here he began his study of Colombian folk music. This part of Colombia's music is all born of the Andes. Colombia has many different musical traditions that vary from region to region. The music of the Atlantic Coast region of Colombia, which includes Cartagena

\footnotetext{
${ }^{3}$ Varney (145-150)
} 
and Barranquilla, is not represented here. These regions are the birthplace of the Cumbia and Vallenato sounds which are not included in this work. Neither is the music of the eastern plains which bears similarities to the national music of Venezuela.

The Bambuco and the Currulao serving as bookends to the work are directly of African descent. They are both from the Pacific coast of Colombia and are notated in $6 / 8$ in this work. Some debate exists about the original way to notate these airs, ${ }^{4}$ but for the purposes of this suite, $6 / 8$ was chosen by the composer as the rhythmic vehicle to convey these movements to the musicians. The pasillo also exists in Ecuador as does the Currulao. The danza air is akin to the Cuban Danzon.

This work is meant to please both the listeners of jazz and the connoisseurs of Colombian music. Both should feel interested in and welcomed by this music. A person who has an ear for the airs of Colombia should immediately discern these airs in this suite. To facilitate that, the guitar becomes the bridging instrument between these distinct genres. It serves to evoke the rhythms in a more familiar fashion for the Colombian listener. Although the fingerstyle guitar is almost exclusively played in this music, the use of a plectrum recalls the work of the bandola player in Colombian music. The typical instrumental ensemble in Colombian folklore consists of a classical guitar playing the bass lines and punctuating the harmony, a tiple, which is a twelve stringed instrument in charge of rhythmic impulse via repetitive syncopated alternating strums, and a bandola. The bandola has sixteen strings, is played with a plectrum, and is in charge of the melodies. It is a cousin of the mandolin from Italy, and the bandurria and laud from Spain's string family. In the author's conception, the alto saxophone and the electric

\footnotetext{
${ }^{4}$ Varney $(80)$
} 
guitar (when playing single lines) replace the bandola, the tiple's role is shared by the cymbals and the guitar (when playing chords) and the piano and string bass play the classical guitar's harmonic and rhythmic foundational role. Highlights of this style include the work of "El Trio Morales Pino", which is a seminal ensemble of Colombia's music.

Typical vocal writing in Colombian music is scored for two voices, one of slightly higher register than the other singing in harmony. Generally, alternations of 3rds and 6ths are used. However, as counterpoint dictates, occasional use of other intervals is needed two conserve the integrity of both lines. Throughout the work the guitar and alto sax will alternate the lead voice role. This maintains the tradition of the duet from Colombian folklore and makes use of the interesting blend between these instruments.

An aspect that may cause difficulty is the phrasing of jazz lines in triple meter. Harmonic goals are reached a beat sooner than in common time, so the improviser must be able to adjust his phrasing to satisfy the resolutions. The Vals is treated as a jazz waltz, which may prove more familiar to a jazz player, but the other airs are less common, particularly the Pasillo. 


\section{Chapter 2}

\section{BAMBUCO}

This is the air that Colombians of the central region most identify with. It has roots in the Cauca region but quickly made its way around the whole Central Cordillera (Ridge) of Colombia. Along with the currulao, it is considered an Afro-Indian product that made its way into mainstream culture in the mid 1850 s. It is a notated music, and its performance required virtuosity, so it acquired the status of art music. On the other hand, it also maintains folk traditions of being played by ear on instruments of local design and construction. This dichotomy is a subject of study of many ethnomusicologists.

In this suite, Bambuco is a 56-bar composition in three sections that starts out with a walking bass line delineating the initial tonality of $\mathrm{C}$ minor. Its impulse connotes the cadence of the bambuco utilizing alternation between $6 / 8$ and $3 / 4$. The drums and piano enter simultaneously with the bass setting up an introduction using Cminor7 and Abmajor7 for two bars each. The author had several choices for notating this song, but $6 / 8$ proved to be the clearest. The guitar enters on the second pass of this introductory section. (Instrumental introductory sections are ubiquitous in Colombian music. Each song has an instrumental introduction that was written specifically for it.) The author chose to put the melodic interest of this introduction in the string bass voice to state a jazz presence. This is not to say that original Colombian music is devoid of string bass. The original composers of this music were classically trained and wrote for various string ensembles. But in the folk tradition, which the author tried to maintain and expand upon, 
string bass use is sparse. The ideal tempo for this song is dotted quarter equals 100. This provides it with a solid rhythmic direction but keeps it from straying into the Pasillo Fiestero tempo that follows in the fifth movement.

The A section begins with a melody beginning on the Eb which is the third of the Cminor7 chord. The melody is harmonized from below by the guitar using successive thirds and fourths, resolving in 6ths. The quarter note, eighth note alternation of the melody is in keeping with Varney's list of rhythmic fragments, but also connotes the swung eighth notes of Jazz 5 . It was the composer's intention for this ambiguity of rhythms to be noticeable. The melody alone could work with a walking bass line and drumming in $4 / 4$, medium swing time. In this treatment it drives the $6 / 8$ pattern set up by the bass and drums. In terms of its notes, the melody is in stepwise motion with note repetition. It contains an alternating succession of thirds and ninths on strong beats 1 and 4 (in this "Bambuco" the first and fourth eighth note of each measure gets accented) over the Cminor7 chord. At the third bar, which marks a chord change to Abmajor7, the melody accentuates the third of the chord in bar 3 and the major seventh in bar 4 . (Considered by jazz theorists to be "guide tones", tones that most strongly delineate a certain harmony)

Bars 1 and 2 of the A section are duplicated in bars 5 and 6 . At bar 7 and 8 a dominant F9 chord is introduced again as an allusion to jazz. (The dominant IV function is rare in Colombian music.) Bar 9 begins a stepwise descent in the bass line aiming for a half cadence (V7) at bar 12. The melody takes a very typically Colombian turn in these final four bars of the A section. This makes the A section of Bambuco, two 12-bar

\footnotetext{
${ }^{5}$ Varney ibid.
} 
sections which in essence makes it a blues-influenced form. (The 12-bar blues is a

common form played in jazz, consisting of a statement, its repetition with mild harmonic variation, and a counterstatement each of which lasts four bars) The section repeats with the second ending resolving to Cminor7 on the downbeat of the second bar 12 of A. (See Example 2.1)

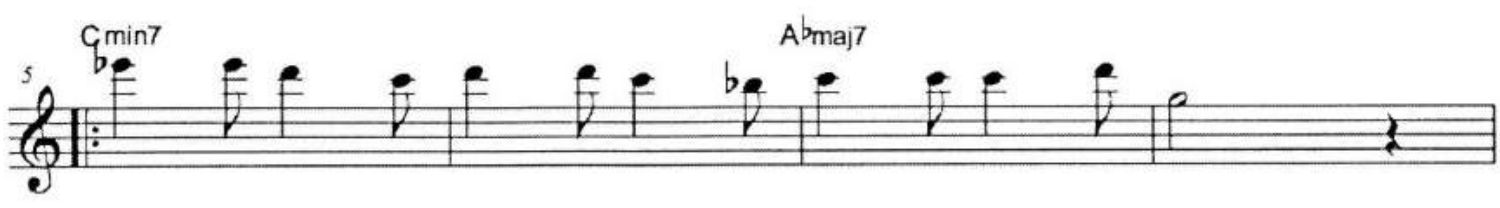

Example 2.1: Melodic excerpt from Bambuco (mm. 5-8)

The B section actually begins in bar 12 of the previous section. On beat 2 of this bar, the Bminor7 chord serves as a chromatic lead in from Cminor7 to the Bbminor7 chord that opens the B section. This is typical of jazz progressions that employ "sidestepping" which is the harmonic deviation by a half step of a progression. This provides some possibilities for sequential development of line when improvising over this part of the song. The tonality now moves to Ab major, using ii7, V7, I major7 and IV major 7 chords in progression. This cyclical chord movement is typical of Colombian music and Jazz music and marks the first four bars of the B section. The melody begins on the $\mathrm{Db}$, which again is the third of the chord. The next bar has some Jazz interest, employing the natural thirteenth followed by the flat $9^{\text {th }}$. This Dominant 7 , flat 9 , natural thirteen chord is atypical of Colombian harmony, but very common in Jazz. This gives way to the first of several stepwise chord movements that appear in the suite. This one ascends from Fminor7 diatonically to the seventh bar which contains a $\mathrm{G}$ major triad over an A bass note. This distinctly mixolydian sound has D major for a parent scale, which is 
set at the distance of a tritone from the original key of $\mathrm{Ab}$ in which the $\mathrm{B}$ section begins. The use of slash chords, the tonicization of mixolydian, and modulation by a diminished fifth are all jazz gestures that mark the B section as the beginning of a departure from traditional Colombian music and a gradual shift into the Jazz idiom. The second time through the section the eighth bar again sidesteps to the $\mathrm{Ab} / \mathrm{Bb}$ chord which leads in to the $\mathrm{C}$ section. The $\mathrm{B}$ section is thus, a sixteen bar section divided in two eight bar phrases. (See Example 2.2)
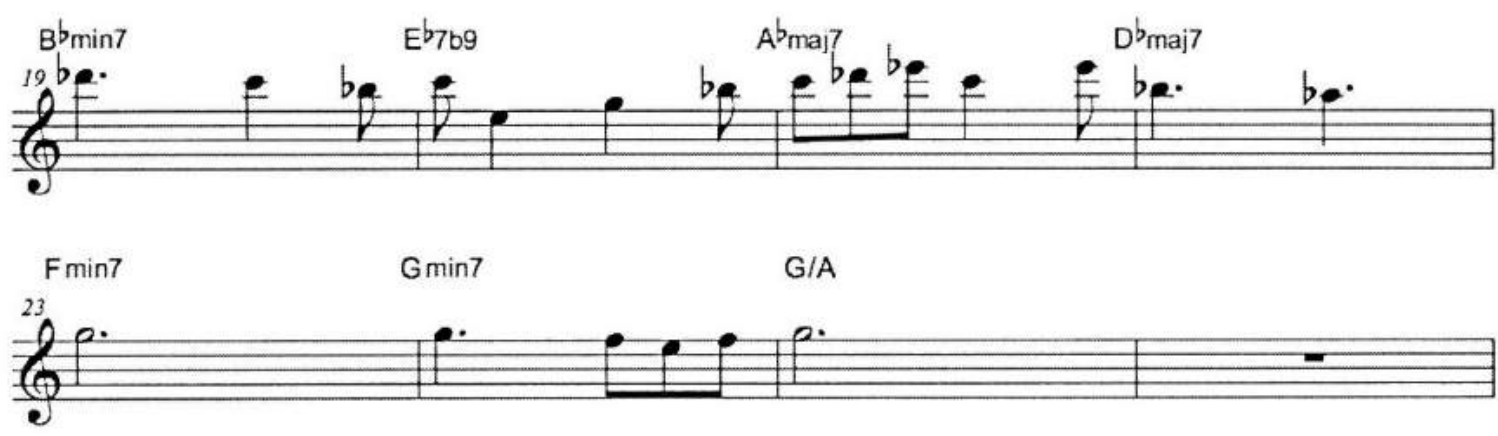

Example 2.2: Melodic excerpt from Bambuco, B section (mm. 19-26)

The $\mathrm{C}$ section is launched by the previous bar. The $\mathrm{Ab} / \mathrm{Bb}$ chord, which has $\mathrm{Eb}$ major as its parent scale, resolves to $\mathrm{C}$ major. This makes for root movement of an ascending second while the actual key is shifting downward by a minor third. This temporary arrival to $\mathrm{C}$ major is brief but effective. It is common of Colombian songs to modulate to the parallel major. It also marks a clear entrance into the $\mathrm{C}$ section. The first eight bars of the $\mathrm{C}$ section are written in four distinct modes, $\mathrm{C}$ Ionian, Eb Lydian, $\mathrm{E}$ Aeolian and A Dorian. The last two share a common parent scale of $\mathrm{G}$ major. (See Example 2.3) 

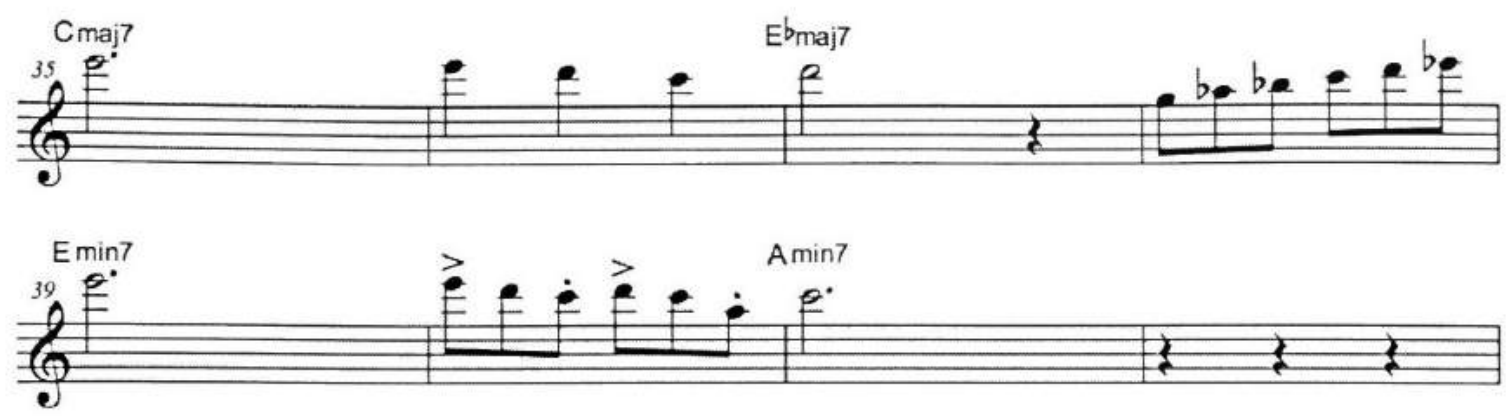

Example 2.3: Melodic excerpt from Bambuco (mm.35-42)

The melodic usage is simplified in this section to allow for more rhythmic embellishment in the rhythm section. The final eight bars of the song are a harmonic sequence of the same progression and melody, first in A minor resolving to $\mathrm{F} \#$ minor (again a parallel relationship) and second in $\mathrm{C}$ minor during the last four bars of the song which draws the idea full circle since the song then begins again in this same key. 


\section{Chapter 3}

\section{PASILLO}

This air is the Colombian version of the $3 / 4$ Viennese waltz tradition. It is however inverted rhythmically. The traditional waltz has one bass note lasting a dotted half note, punctuated by two chord strokes of a quarter note each. The pasillo juxtaposes this pattern placing two bass notes on beats one and two followed by a single chord punctuation on beat three. As was stated in the introduction, the pasillo is shared by Colombia and Ecuador. In Ecuador however, the harmony is slightly different. It has a more pentatonic sound and is more likely to hover around minor keys. The Colombian pasillo is more diatonic and appears in major keys equally as often.

Pasillo as it appears in this suite is a 46-bar form, divided into an eight bar introduction, a 16-bar statement made twice, with a 6-bar coda. It maintains a typically Colombian harmonic rhythm, but the band implies a modern urban funk sound in $3 / 4$ time. The original air is still discernible, implied more by the form of the song and by the melodic choices made than by the rhythm section. The introduction or prelude is in C\# minor. The key movement of the main body of the song is organized in Coltrane's Giant Steps cycle, appearing here in the Db, F, A key succession. (The original uses the B, G, Eb system) This system of keys denotes an augmented triad when its roots are stacked harmonically.

An Amajor7 with the sharp eleventh in the melody is the first sound heard. This begins an eight bar section which serves as the "instrumental" introduction to the song. 
The root movement in the first eight bars is typically Ecuadorian. The beginning of phrases on the VI chord of the minor key is most common in the pasillo of Ecuador. The VI, III, V7, I minor is seen in various Ecuadorian pasillos. The melody is typically Andean except for bar 5 , which has a sharp ninth. This has very little usage in Colombian pasillo, but occasionally one hears a guitarist play a $\mathrm{C}$ major triad, against an $\mathrm{E} 7$ chord in Ecuadorian pasillo thus unwittingly implying an altered sonority. Pentatonic melodic writing is also present for the first time in the suite. (See Example 3.1)
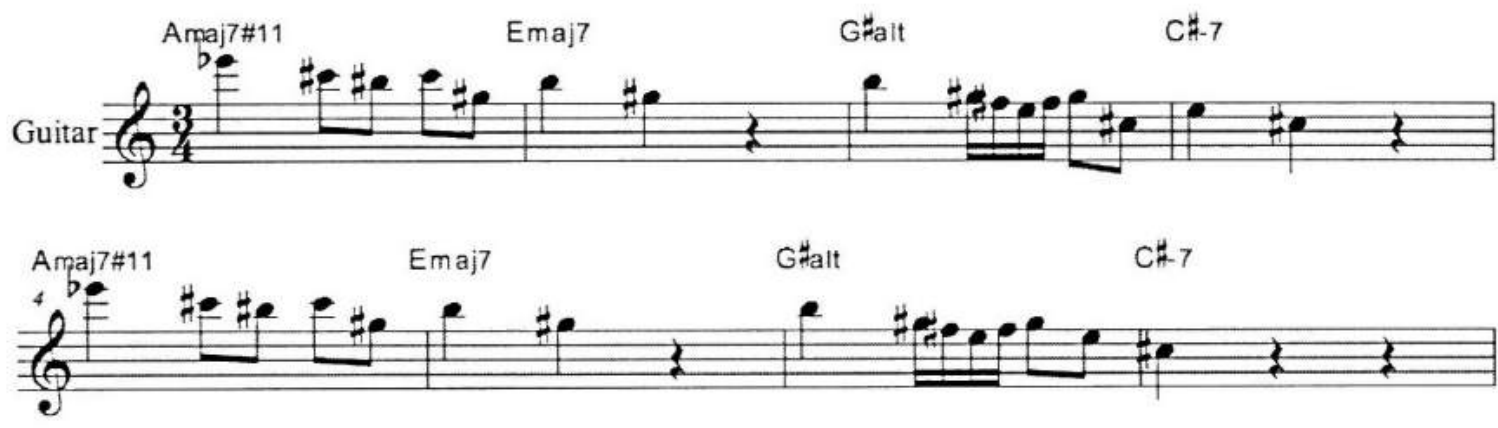

Example 3.1: First eight bars of Pasillo

The body of the song begins in bar 9 . The melody begins with a tritone leap from the flat ninth to the fifth to imply a dominant chord during the stop time leading into the Ebmin9, which is topped by a ninth. This is followed by a Gb minor(maj7) resolving to a $\mathrm{Db} / \mathrm{F}$ chord thus completing the first clear statement in $\mathrm{Db}$ major. This arrival only lasts a bar. The subsequent E13 chord marks a key shift of a major third in descent, starting the Coltrane cycle with a modulation to A major. A ii- $\mathrm{V}$ progression ensues in bars 5 and 6 followed by a distinctly Jazz influenced moment in bar 7 where there is an accented major ninth put on top of a C\# half diminished chord thus invoking a Locrian \#2 mode, which is from the E melodic minor parent scale system, another common jazz usage that is not at all associated with Colombian music. This is followed by an F\#7 chord which 
makes for the second un-resolved ii- $\mathrm{V}$ progression within four bars. The melody in this song does not contain many large leaps and is basically contained within an octave, some of which are traits common to Colombian pasillo melodies. The beginning of the theme takes place on the up-beat of the first beat of the measure. This is most common in pasillos and valses (waltzes) of the Colombian and Ecuadorian folk tradition. Also there are repeating notes in the melody typical of songs with lyrics. Throughout this work, the author had certain textual ideas regarding the would-be subject matter that these songs would set well to. This song reflects that most of all. It could be easily adapted into a vocal melody. (See Example 3.2)
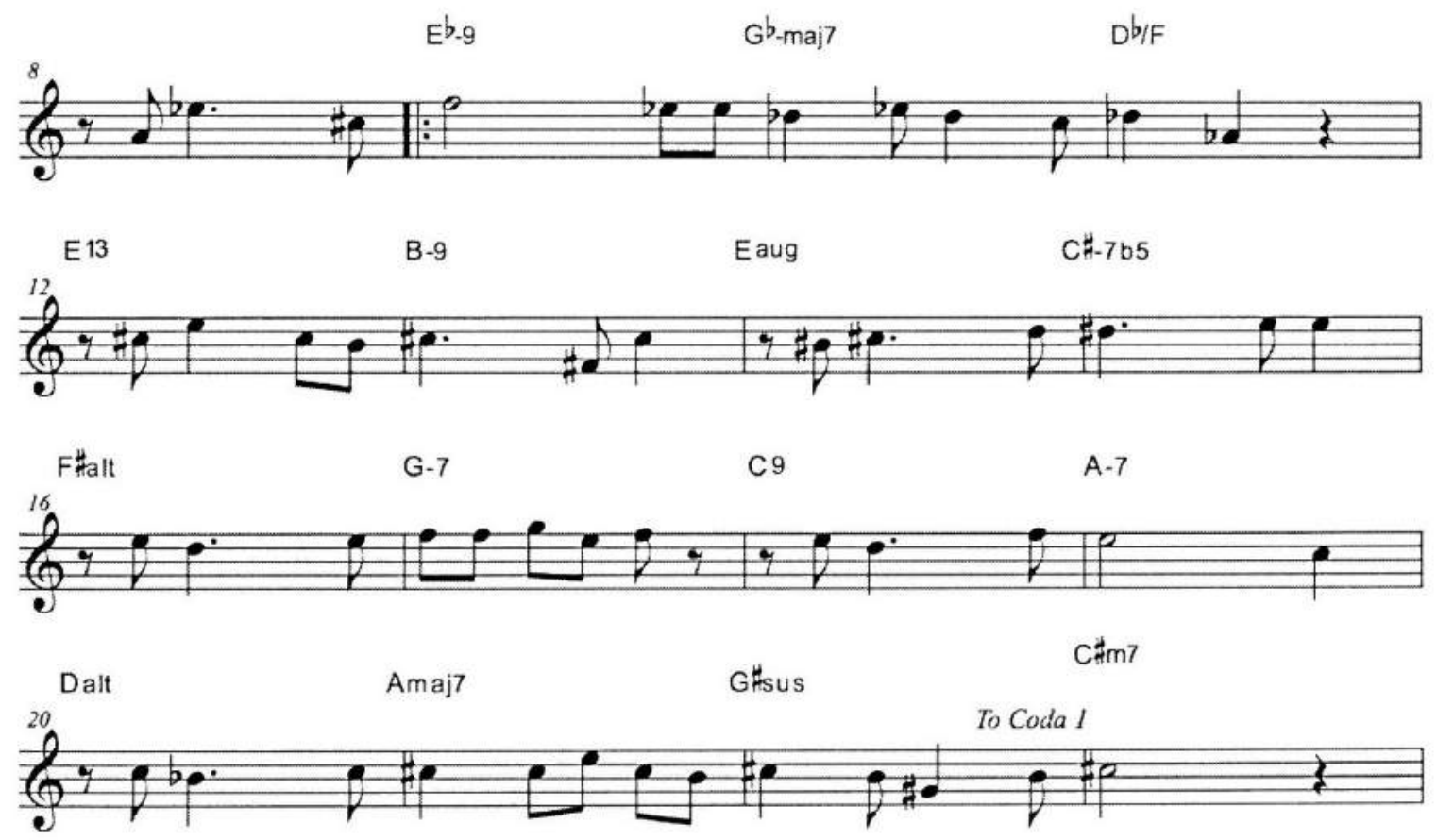

Example 3.2: Melodic excerpt from Pasillo (mm. 8-23)

Bar 9 starts a new series of unresolved ii-V progressions in the final key of $\mathrm{F}$ major completing the Coltrane cycle of keys. This is the climax point of the melody. From here the song returns to $\mathrm{C} \#,(\mathrm{Db})$ for a pentatonic ending. The second time through there is a 
6 bar tag added that contains the second of the suite's progressions with root movement consisting of seconds. (See Example 3.3)

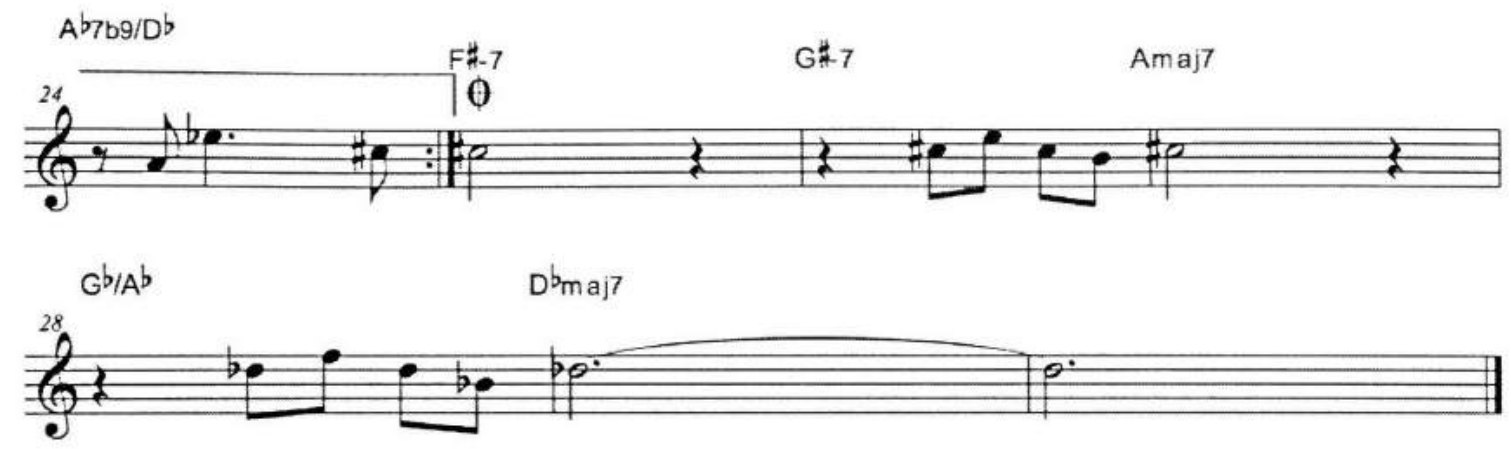

Example 3.3: Ending of Pasillo 


\section{Chapter 4}

\section{DANZA}

The danza rhythm is the only rhythm in the suite that is duple. It is a descendant of the Cuban Habanera and certain dances of Spain, which also spawned the Cuban danzon, and possibly the Latin bolero. Its strains can be heard throughout Colombia, but most especially in the Valle region, where there are hundreds of obscure danzas that are all from an area of about 30 square miles, another characteristic of folk music; its regionalism, how slight geographical differences can mark distinct changes in style and esthetic.

This 25 bar song, with an eight bar intro, is the most modern composition in the suite. Using the least syncopated air in the work, Danza has an initial melodic statement, which is made up of all twelve notes available. (The Currulao will also have a twelvetone line, but not harmonized) Via a series of triads superimposed over an A pedal point, the author managed to harmonically support each gesture of the line, in so easing the possible atonal tension that a dodecaphonic melody would be expected to create. This melody is not technically a tone row, because the $\mathrm{F}$ is repeated. The line has several triplet figures, which serve to soften the angles of the melody. The melody is also designed in a fashion that allows it to be not rhythmically strict. The alto saxophonist and guitarist who carry the theme in unison, simply need to follow each other in order to maintain a coordinated rubato ensemble sound. The twelve tone statement is repeated with bars 7 and 8 containing the first deviations from the pedal point found in the opening bars, a C\#minor9 chord and a Bb6 chord, respectively. (See Example 4.1) 


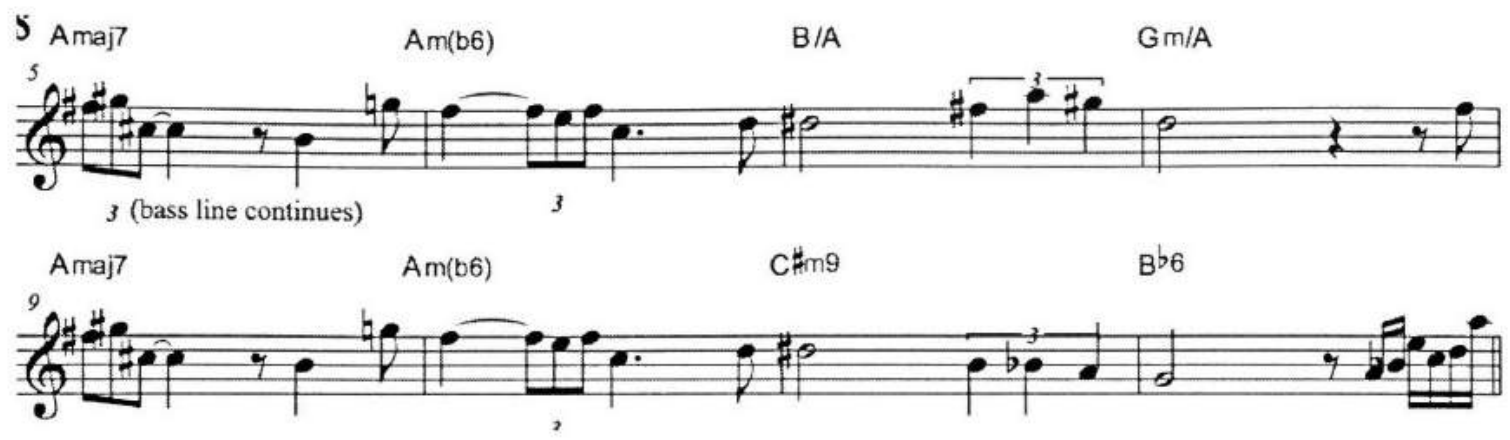

Example 4.1: Opening melodic statement of Danza (mm. 5-12)

The second leg of the song starts with the most rhythmically active melodic statement yet heard in this movement, a sixteenth note flourish on A phrygian, which when set against a harmonic backdrop of $\mathrm{Bb} 6$ creates a distinct $\mathrm{Bb}$ lydian sound. The final $\mathrm{D}$ concert note of the sixteenth note run is the first note of the $9^{\text {th }}$ bar, which is the beginning of the second section. This note is harmonized by a Bminor7 chord, which changes the character of the piece and initiates a slight turn forward in rhythmic intensity, promoted by the sixteenth note passage. The third line of the piece has two triplet statements on B minor and then $\mathrm{C}$ minor. This half step shift builds via dynamics to a series of slash chords (triads superimposed over non-harmonic bass notes) that mark the songs first moment of arrival. This is the loudest part of the piece thus far. The scales implied by these chords are: D melodic minor, F\# Phrygian, G auxiliary diminished, and finally $\mathrm{Bb}$ major which is the release of this climactic gesture. (See Example 4.2)

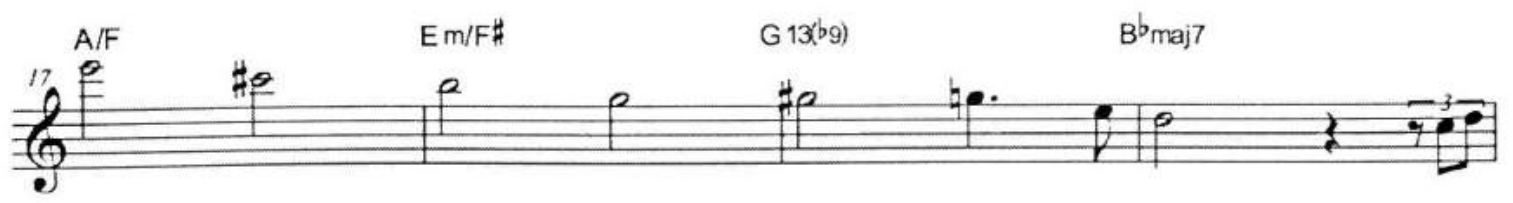

Example 4.2: Melodic excerpt from Danza $(\mathrm{mm} .17-20)$ 
These four bars should not only be pivotal in the dynamic curve of the piece, but the should themselves be imbued with an arc building to bar 15 with the G13b9 chord and sinking back to a placid mezzo piano on the Bbmajor7 chord. Then the final leg of the song begins in bar 17 with yet another stepwise progression from, Dminor7 to Ebmajor7(\#11) and then F major finally ending with a descent to a Db half diminished chord which serves as a plateau to set up the final phrase. (See Example 4.3)

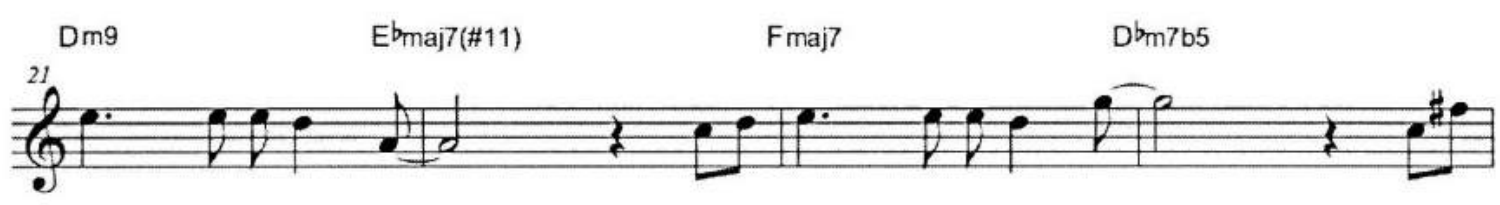

Example 4.3: Excerpt from Danza (mm. 21-24)

Three consecutive major chords, Cmajor7, Bbmajor7 and Ebmajor7 frame the final ascent to a B phrygian chord. The line rises in pitch gradually to an E concert at the beginning of bar 24 . Here the author placed one of the many blues figures that are scattered around the suite, which the band accentuates together at a high intensity. Then the bass returns with its written ostinato outlining the original chords that introduced the movement. (See Example 4.3)

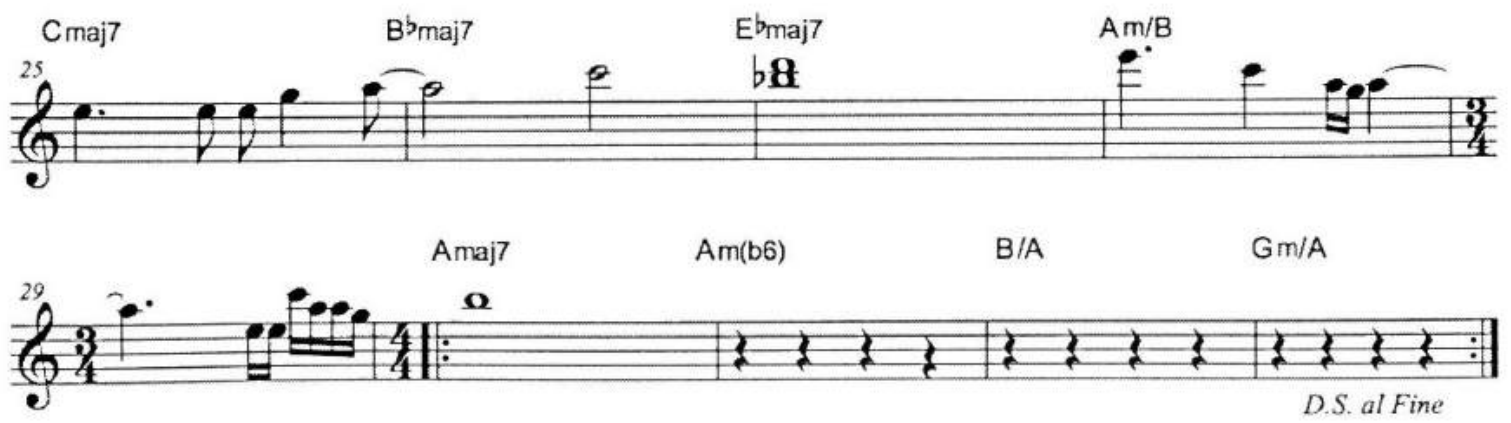

Example 4.3: Melodic excerpt from Danza (mm.25-33) 
In terms of improvisation, this is the most exacting progression to negotiate that can be found in this work. Every bar line requires a harmonic alteration of scale (Bars 11 and 12 could perhaps both be played over in $\mathrm{C}$ aeolian). It is the most challenging vehicle for a soloist in this suite. The other pieces have some phrasing issues that are exacerbated by the triple meters, but this one has many harmonic details that have to be taken into account to successfully improvise over its tonal changes. 


\section{Chapter 5}

\section{VALS}

As mentioned in the second chapter, the Viennese waltz influenced the writing of Colombian composers. It was undoubtedly brought to Colombia in the European migration. It is the only air in the work that is unaltered rhythmically by New World musicians. It maintained its cadence in Colombia, and made its way to Peru and Argentina where beats 2 and 3 are treated in a more staccato fashion.

There is a strong waltz tradition in jazz as well, so the author chose to give this piece a clear jazz waltz feel. This also helps differentiate it from the other triple meters in this work. It is the most light-hearted movement in the work, marking a departure from the subdued intensity of the Danza. It is not without harmonic interest and detail but in spirit, it is supposed to be somewhat jovial. Hitherto, the pieces have had enough tonal shifting to make any reference to them being in a certain key inaccurate, but the Vals is strongly centered in D minor. (The Bambuco only spends 16 bars in Cminor out of 56) The eighth notes should be played inegale or swung, promoting a triplet feel, and the dynamic remains mezzo forte to forte. It consists of a 32-bar section, split into two 16 bar sections. It ends with a 16 bar section, eight of which are spent in D major and the other half in Fmajor/Dminor.

Many Colombian waltz and pasillo melodies start on the upbeat of beat one. This tradition was continued in the Vals and the Pasillo. The melody starts with a phrase again taken from pentatonic, on Dminor7. The Ab melody note on the downbeat of bar 2 comes from the D blues scale but $\mathrm{I}$ harmonized it in Db major again in keeping with the 
sidestepping tradition of jazz composers. The minor third descent to Bbmajor7 reinstates D minor as the harmonic canvas for the piece. The first statement ends with a stepwise chordal ascent from Fmajor7 to Gminor7 to G\# fully diminished, resolving in A7(b13) all of which are harmonically in line with the D minor tonality of this movement. The first four bars repeat in bars 9 through 12. Bar 13 marks the first and only lengthy departure from the tonality of $\mathrm{D}$ via a 4 bar ii- $\mathrm{V}$ progression in the key of A major. The A melody note that served as the third of $\mathrm{F}$ major in bar 5, is taken up an octave in bar 13 and serves as the seventh of the Bminor7 chord serving as ii- 7 in this portion of the tune. (See Example 5.1)
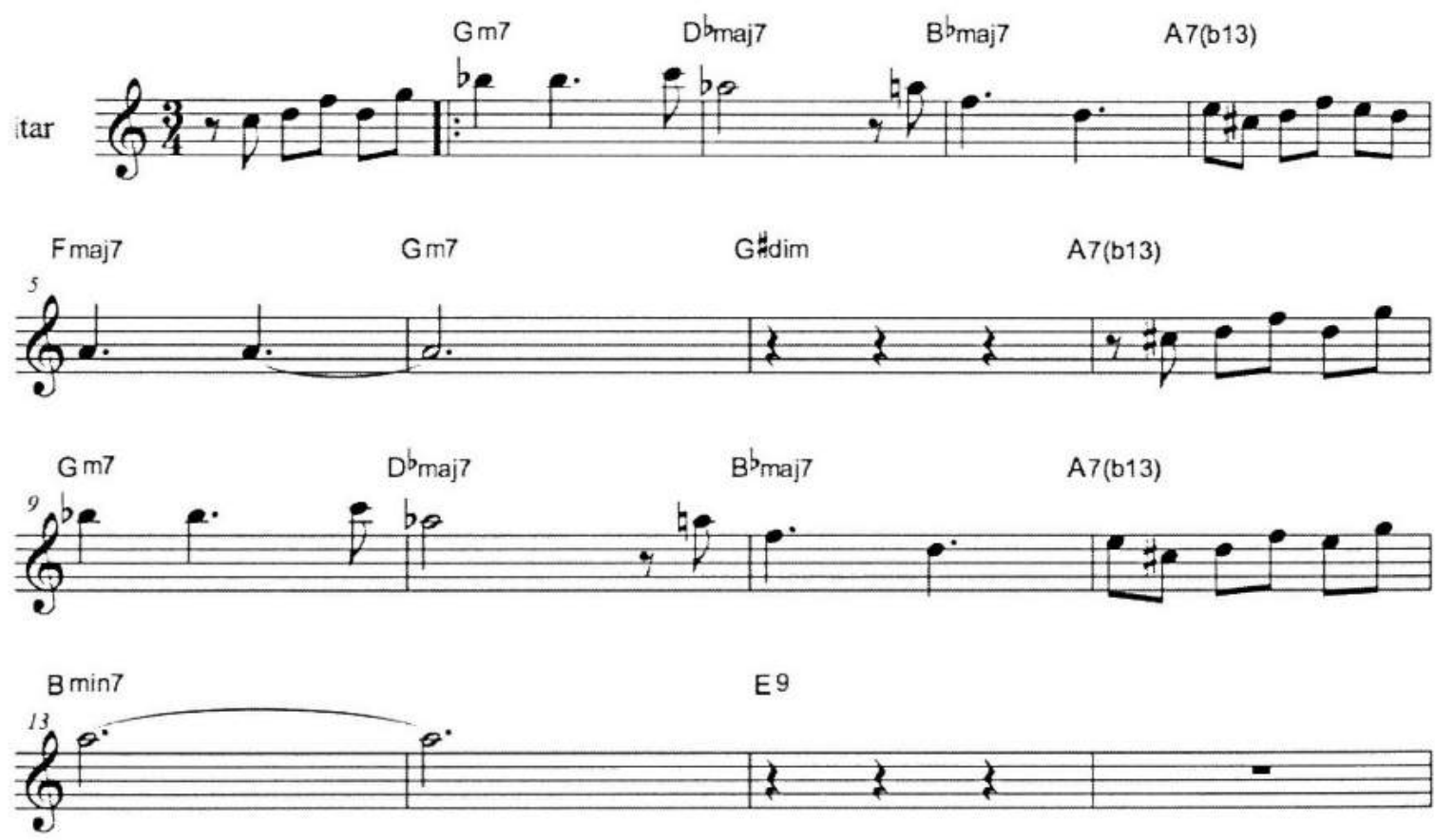

Example 5.1: Opening melodic statement of Vals (mm. 1-16)

Bar 17 begins a stepwise progression that lasts through 16 bars. B half diminished, Bbmajor7, Asus7, Abmajor7 (\#11), Gminor7 and Fmajor7 all come in succession while the melody repeats the same figure twice with some alteration to satisfy 
the harmony. Ending this section is a Two-Five progression in D major. The author wrote a $\mathrm{C}$ natural in the melody over a Dminor 11 chord in bar 30 for its character. It implies E locrian and not the usual dorian. (See Example 5.2)
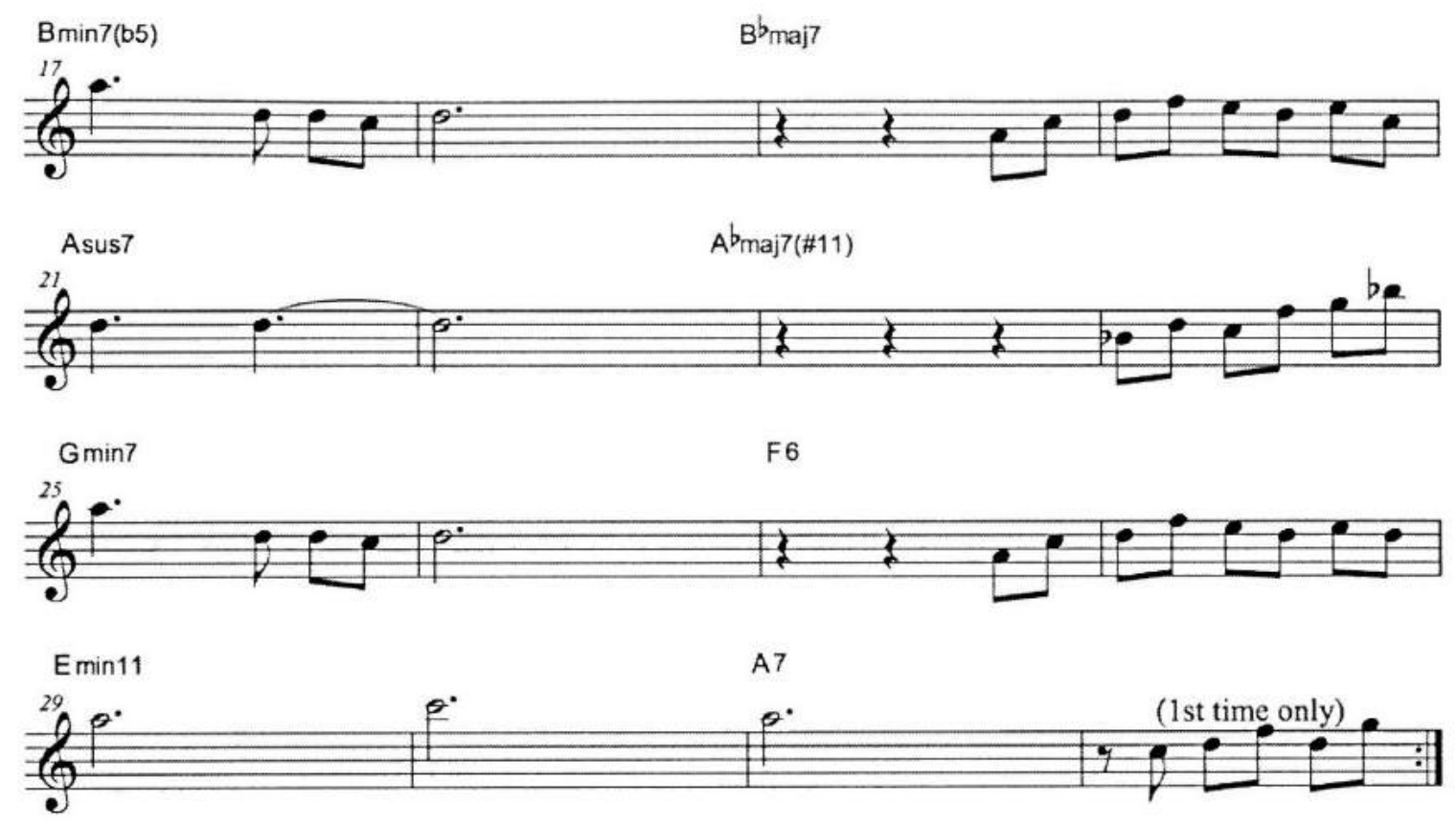

Example 5.2: Melodic excerpt from Vals (mm. 17-32)

Bar 33 marks a departure to the key of D major instigating a distinct character change in the piece. The harmony is diatonic and descends from Gmajor7 to C major7. The melody is an embellished descending D major scale. It is the simplest moment of the suite in harmony and melodic interest.

The final eight bars behave sequentially with the eight previous bars, maintaining melodic contour and a major sonority, but they are in the relative minor of the original key. The piece then recapitulates for the improvisations.

In character this piece and the following Pasillo Fiestero bear the most resemblance to their original counterparts. They have a more similar harmonic make up 
and melodically are traditional in rhythm and note barring a few minor deviations. (See

Example 5.3)
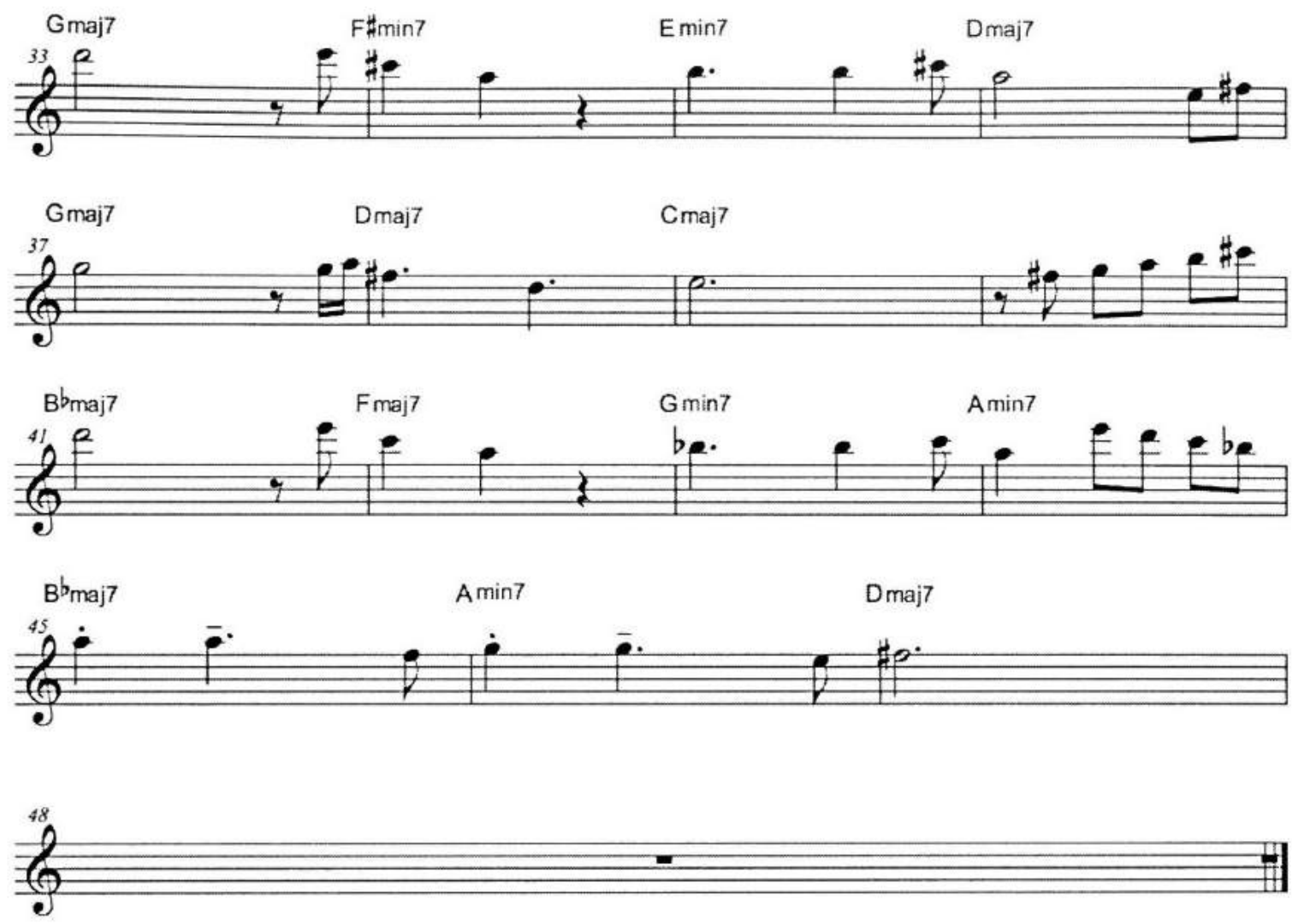

Example 5.3: Final sixteen measures of Vals (mm. 33-48) 


\section{Chapter 6}

\section{PASILLO FIESTERO}

The virtuosic tradition in Colombia has a strong place. Society has perennially valued instrumental dexterity, extolling it in mainstream recordings. There are various national contests of traditional instrumental music such as the Mono Nunez festival in the city of Ginebra in the state of Valle.

In keeping with this tradition, the author composed this Pasillo Fiestero. It is differentiated from its namesake Pasillo by the descriptive Fiestero, which loosely means of a party or festive. This additional descriptive term, when added to a traditional air, simply means the tempo is raised, sometimes dramatically. The optimum tempo for Pasillo is ca. $96 \mathrm{bpm}$. The Pasillo Fiestero quite the contrary clocks in at about $262 \mathrm{bpm}$. This is the typical tempo for this air.

Pasillos of the fast variety are generally interpreted by the bandola. As previously stated, this is a cousin to the mandolin. Virtuosity on this instrument is and has always been highly regarded in Colombia. The "Trio Morales Pino" is the standard bearer for this modality of performance. The author's grandfather, Jose Manuel Bermudez was a well-known performer of this traditional music. This suite is in tribute to him and to the author's father. Most especially this movement is meant to sound authentically Colombian, the only difference being the instrumentation.

Strongly rooted in $\mathrm{F}$ minor, this song starts with a 6-note pickup that sets up the tempo. The first four bars outline the chords clearly using F harmonic minor, which is a 
typical scale in Colombian composition. The Dbmajor7 chord in bar two is uncommon in Colombia only because of the Major seventh. The bVI function is however widely used.

The fifth bar on a $\mathrm{C}$ altered chord, employs the seventh mode of Melodic Minor, also known as the altered scale. This provides for the availability of a minor third on a dominant chord. (The proper name for that interval is the sharp ninth.) This is most unusual in Colombian harmony but it makes for a surreptitious inclusion of the Jazz harmony framing this suite. The third phrase of the A section also includes the altered sound, this time on $\mathrm{F}$ altered which acts like a secondary dominant resolving deceptively to Dbmajor7 again. Then a stop time occurs on Gbmajor (Neapolitan). To close the first section, a back door ii-V progression consisting of Bbminor7 and Eb7 was chosen. This then resolves up a whole step to the F major. (See Example 6.1)

$$
\text { Fmin7 } \quad \text { Dimaj7 } \quad \text { C 7b9 }
$$
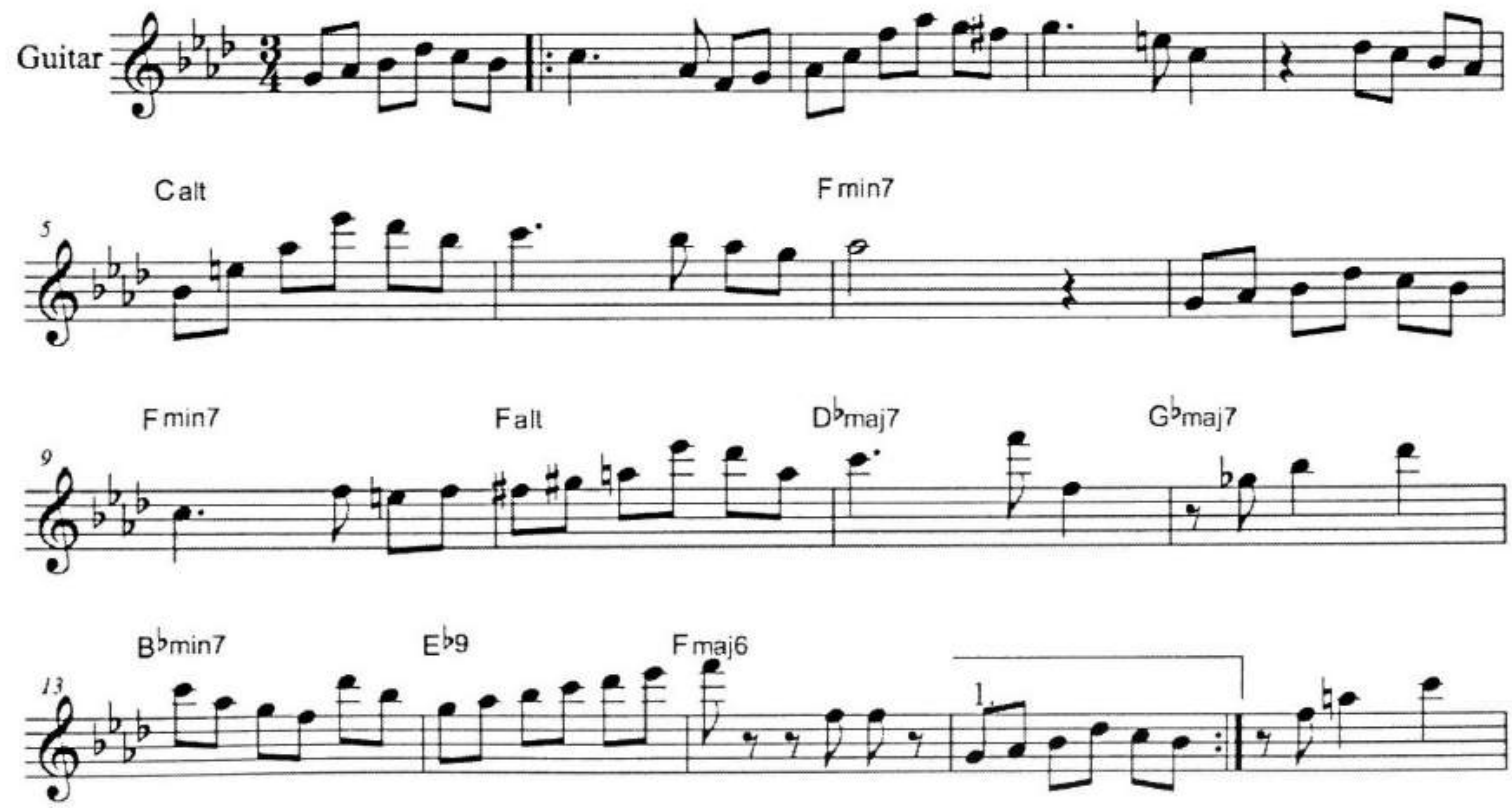

Example 6.1: Opening melodic statement of Pasillo Fiestero (mm. 1-17) 
The B section includes the final stepwise progression and a more jazz oriented melodic approach. This section adopts a slight swing, but it is masked by the fast tempo that evens out the eight notes. (See Example 6.2)
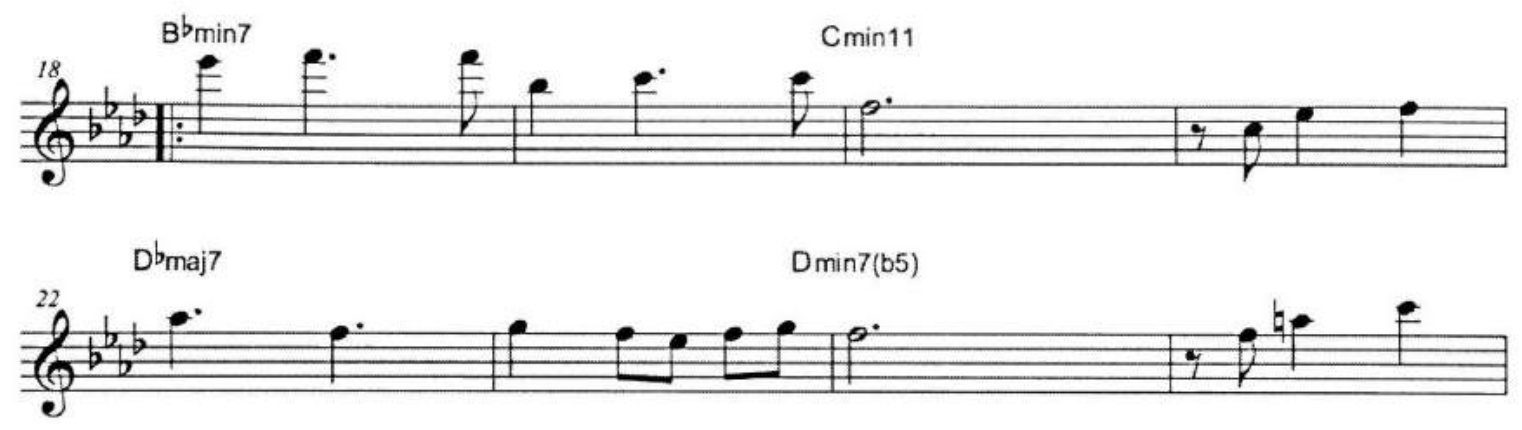

Example 6.2: Excerpt from Pasillo Fiestero (mm. 18-25)

The progression ascends from Bbminor7 to D half-diminished via Cminor7 and Dbmajor7. The improvisation should take place only on this section.

The last section is built melodically on a repetitive pattern very typical of those found in Colombian fast pasillos. The first sonority on this section is however not found readily in traditional Colombian music. The iv half diminished function occurs in true harmonic minor harmony if the raised eleventh is brought down an octave. It is basically a iv-i-V7-i progression. The final chromatic passage is meant as a hemiola felt in $3 / 2$ time. (See Example 6.3)
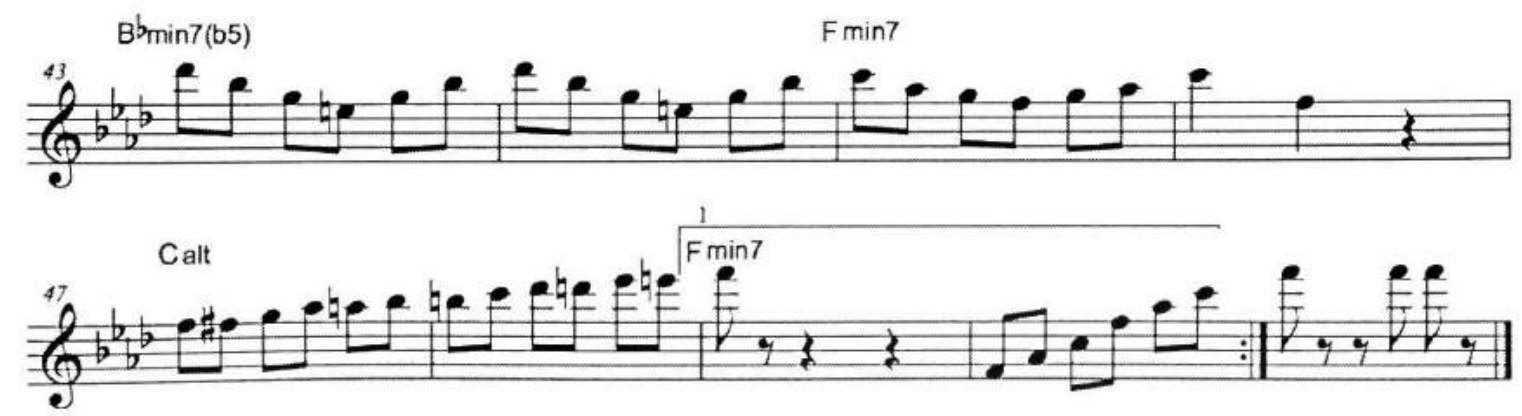

Example 6.3: Ending statement of Pasillo Fiestero (mm. 43-50) 


\section{Chapter 7}

\section{CURRULAO}

The currulao is an Afro-Latin rhythm found mainly in Colombia and Ecuador. It can be notated either in $12 / 8$ or as it appears in the suite, in $6 / 8$. This air was originally interpreted by percussion ensembles. According to Varney, these ensembles generally consist of a guasa (shaker), cununo drums, a bass drum and a marimba for melodic interest ${ }^{6}$. The guitar introduction of the Currulao in this suite imitates the marimba Chocoana, a xylophone type instrument made of wood which is from the Choco state of western Colombia, a predominantly Afro-Colombian area. The instruments each add a part to the rhythm adding quadruplet gestures to the triple meter to provide rhythmic counterpoint and African elements of syncopation and polyrhythm. The intonation of The traditional "caderona" (woman of big hips) call and response chant can be chosen in performance if an adequate singer is available. The band can respond in chorus. This call and response tradition in African music has left lasting effects that are readily noticeable in rural blues, gospel and spiritual songs, jazz, and virtually every popular genre. In this piece it shows traditional influence and evokes the original nature of this air. If lacking a singer the band can enter directly into the written portion of the song.

The saxophone plays the melody alone for the first time in the suite. This melody begins in the high register of the alto sax creating intensity via the urgent sound of this tessitura. There is a tradition of clarinet playing in the southwestern region of Colombia where the currulao originates, most likely due to European influence. The saxophone

\footnotetext{
${ }^{6}$ Varney $(40)$
} 
emulates the clarinet, but provides an added degree of stridence and intensity. This melody is in fact a Tone Row utilizing all twelve tones without repetition through the $\mathrm{Db}$ note that is the flatted $5^{\text {th }}$ of $\mathrm{G}$ blues, the source of this sections ending phrase. (See Example 7.1)

itar
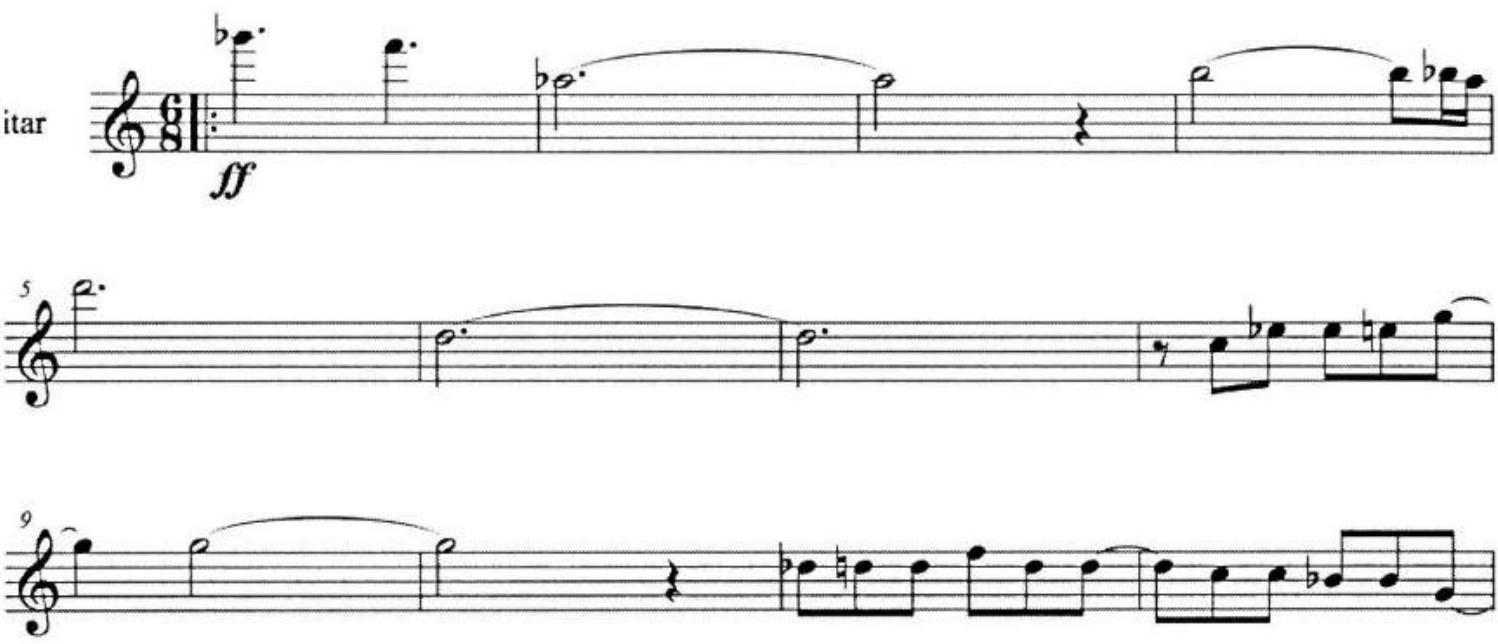

Example 7.1: Melodic excerpt from Currulao (mm. 1-12)

The first three notes $\mathrm{Gb}, \mathrm{F}$, and $\mathrm{Ab}$ neighbor the $\mathrm{G}$ pedal point implied by the bass and guitar. In the ensuing line three chromatic tones precede a D, stated twice consecutively. With the phrase ending on a D note, the G tonality is strengthened. The ensuing phrase when played against a $\mathrm{G}$ pedal implies a blues sound with both natural and flat 13 ths followed by the final phrase that solidifies $G$ as the tonality, via the flatted $5^{\text {th }}$, natural $5^{\text {th }}$, flatted $7^{\text {th }}$, the fifth, fourth and minor third successively.

At this point the rhythm and intensity of the ensemble should be at a peak for the entrance of the B section. This B section is built on G minor. It is repetitive and recalls in its syncopation the sound of the music of Colombia, in this case the Pacific coast, while via this instrumentation maintaining a clear modern jazz esthetic, which again is the concept for this whole work. 
Musically speaking, the melody begins with a minor third interval from $\mathrm{G}$ to $\mathrm{Bb}$ using repetition of the $\mathrm{G}$ to anchor the harmony. The notes come from the $\mathrm{G}$ minor pentachord of G, A, Bb, C, and D. (See Example 7.2)
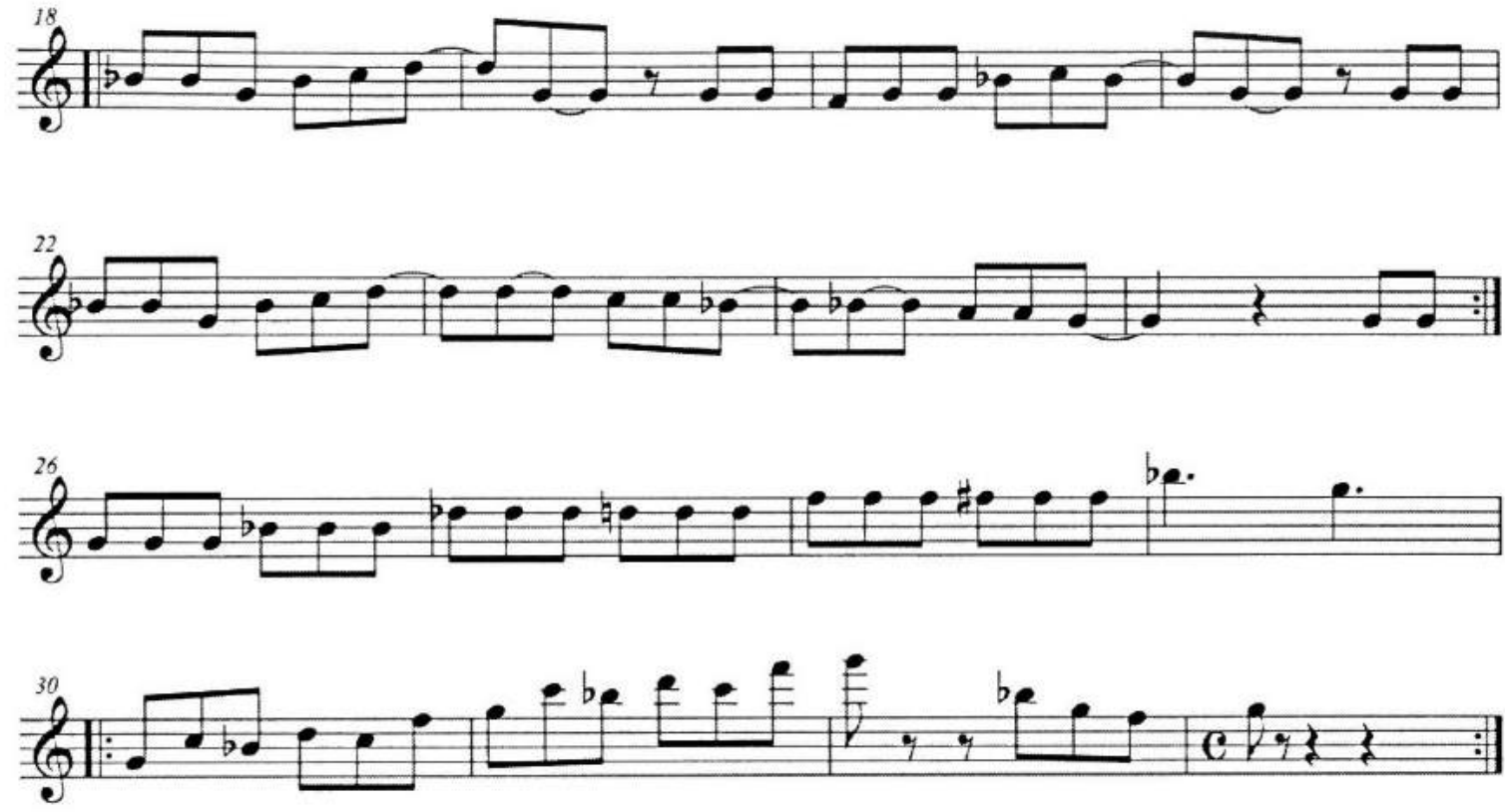

Example 7.2: Excerpt from Currulao (mm. 18-34) 


\section{BIBILIOGRAPHY}

Tirro, Frank. Jazz: A history. New York: W.W. Norton and Co. 1993, 1977

Varney, John. "Colombian Bambuco: The evolution of a national music style. Ph.D. thesis, Queensland Conservatorium, Griffith University, Australia (1999) 


\section{BAMBUCO}

Alvaro Bermudez
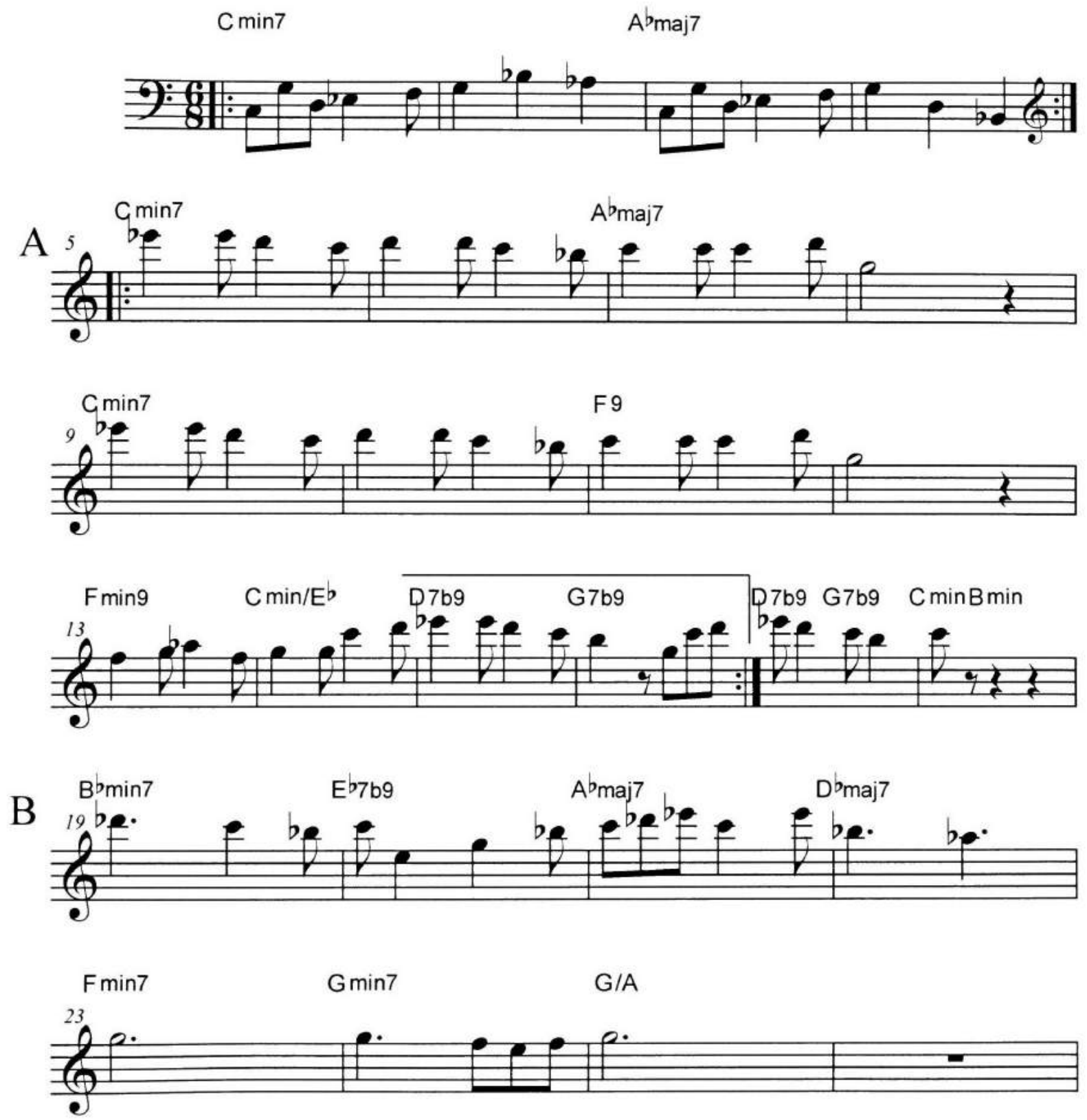
${ }^{c}$
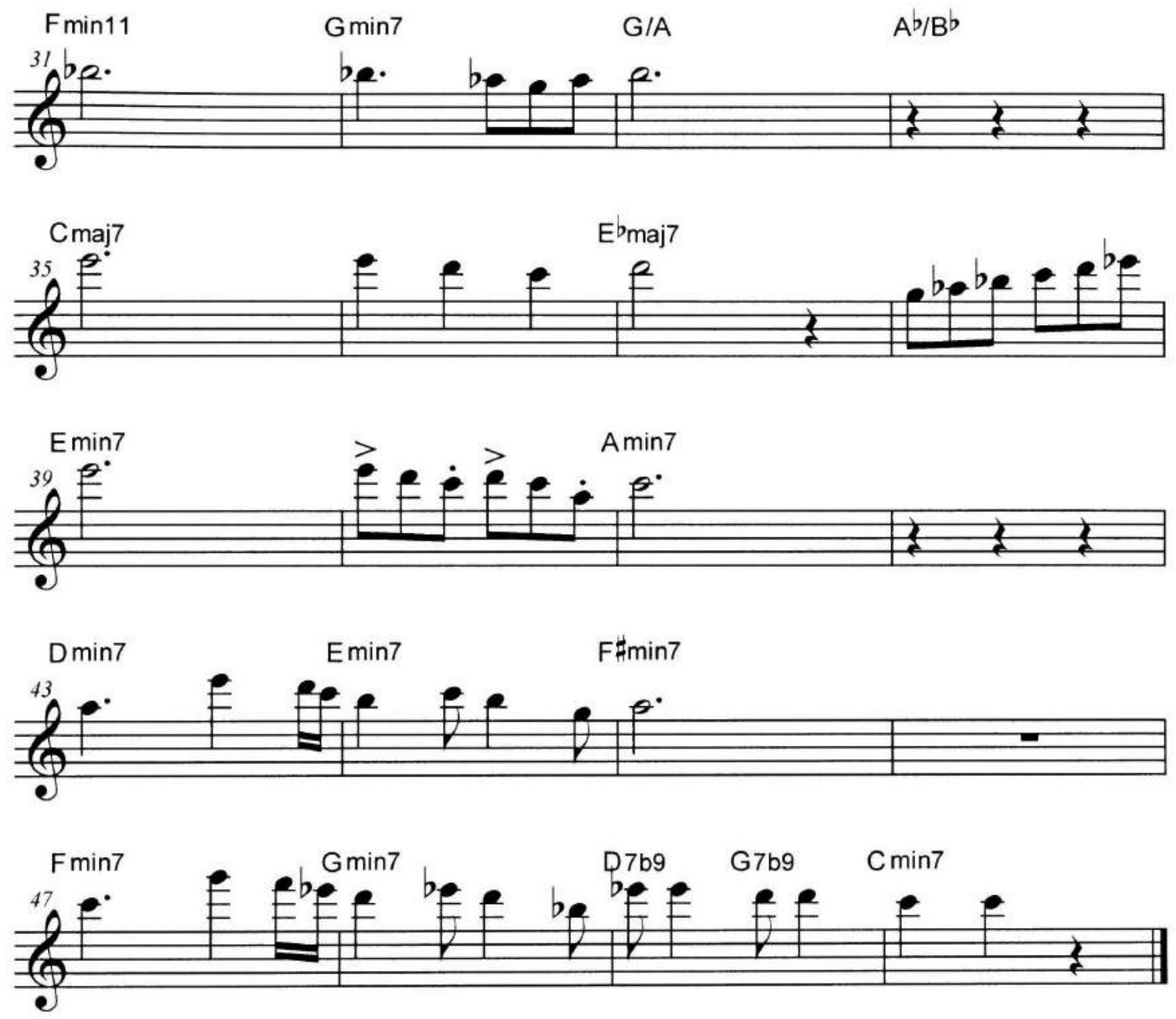


\title{
I. BAMBUCO
}

Alvaro Bermudez

\author{
$A \min 7$
}

Fmaj7
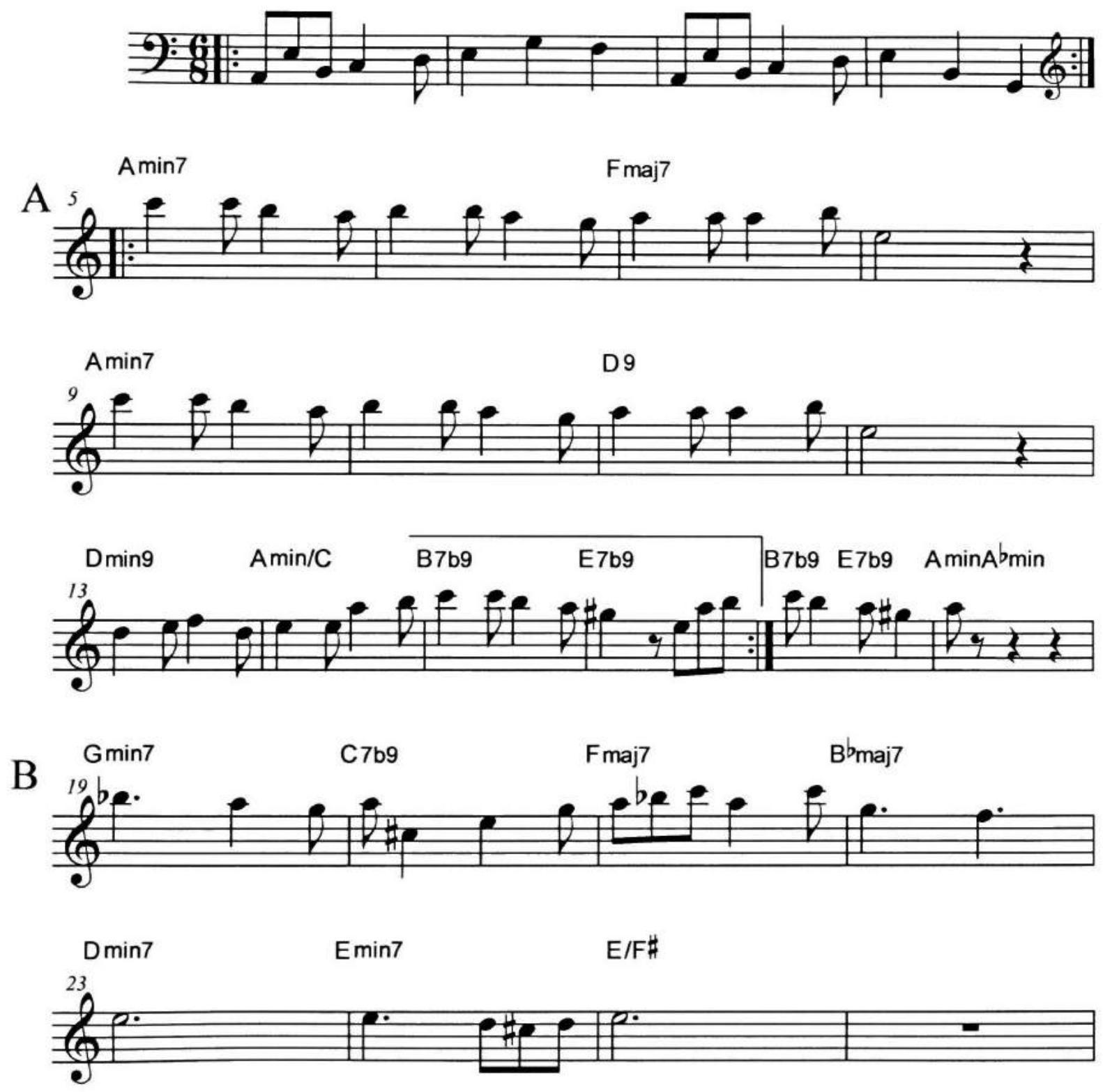

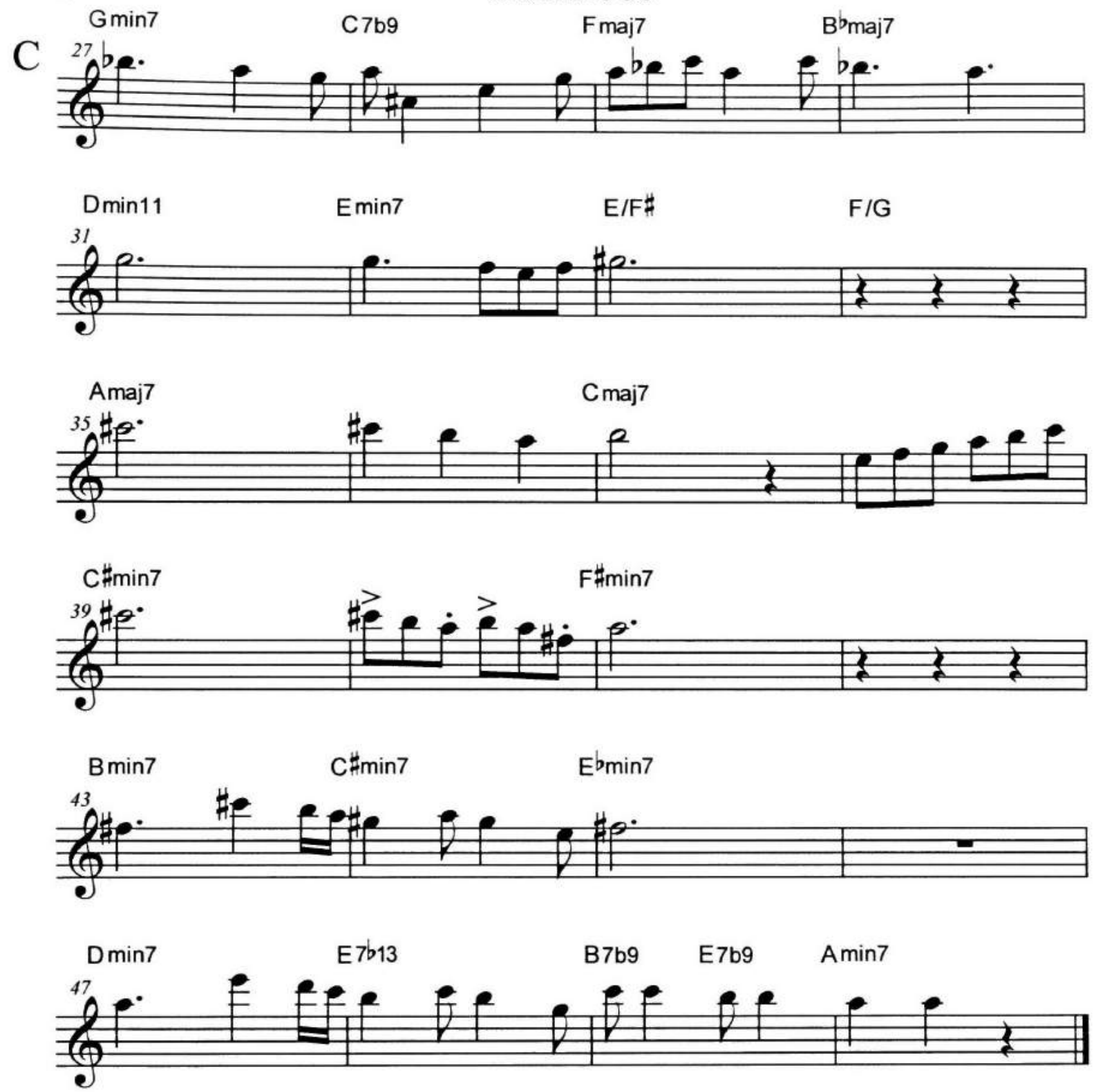


\section{PASILLO}

ALVARO BERMUDEZ
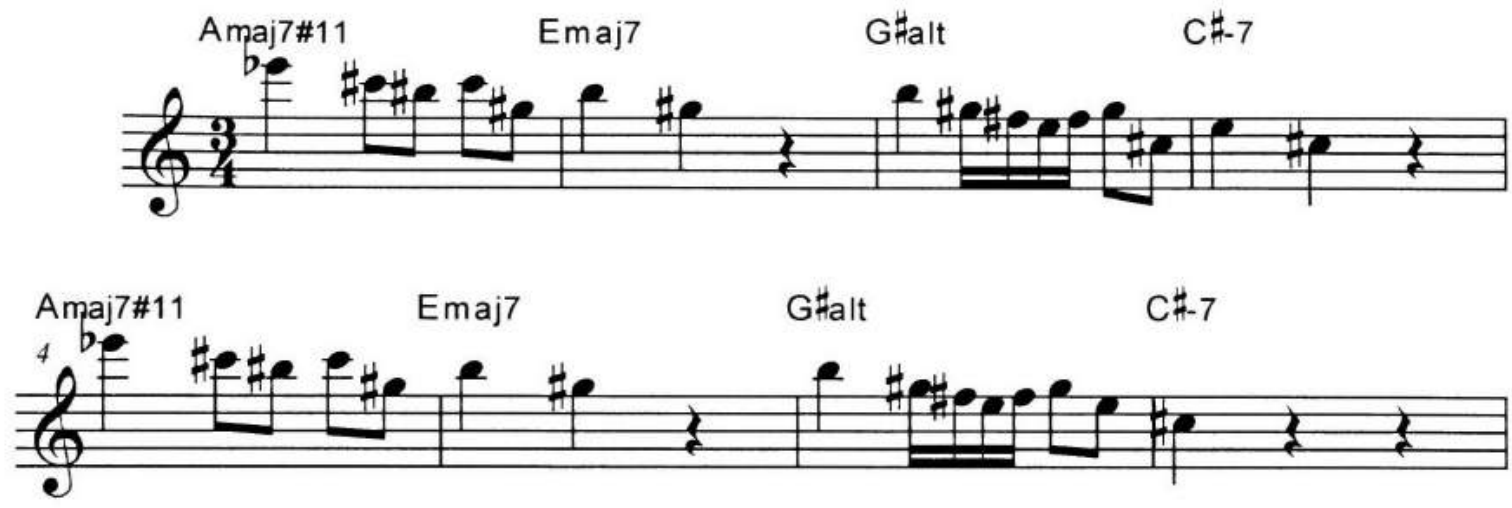
$E^{b-9}$
Gb-maj7
$D^{b / F}$

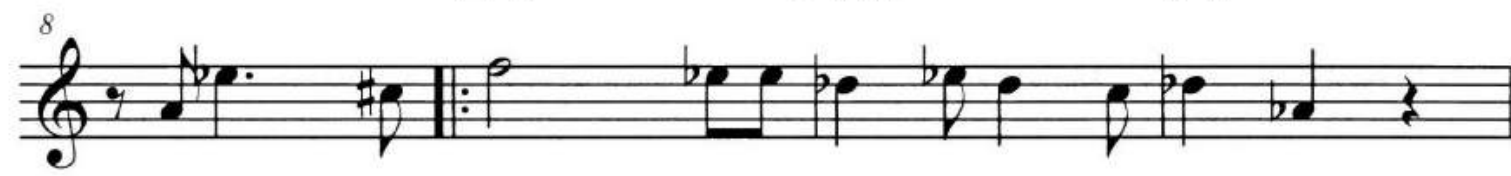
E13
B-9
Eaug
$C \#-7 b 5$

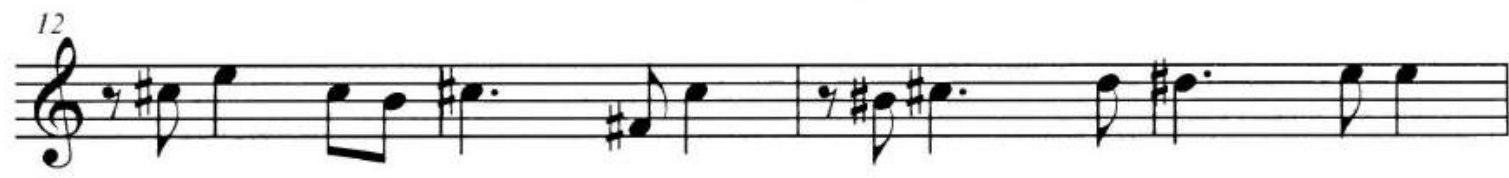
FFralt
G-7
C9
A-7

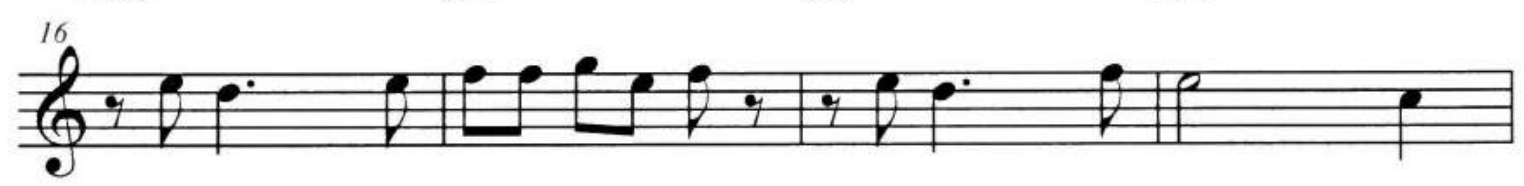
Dalt
Amaj7
G\#sus

$$
\mathrm{C} \sharp \mathrm{m} 7
$$

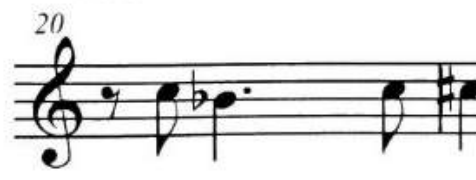

To Coda 
2 Ab7b9/Db

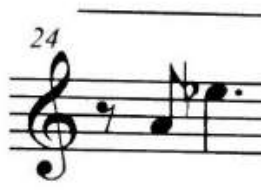

$G^{b} / A^{b}$
PASILLO

F\#-7

$\theta$

$\mathrm{G} \#-7$

Amaj7

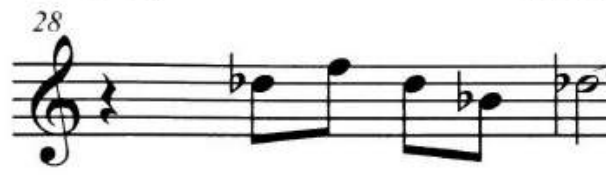

$D^{b}$ maj7
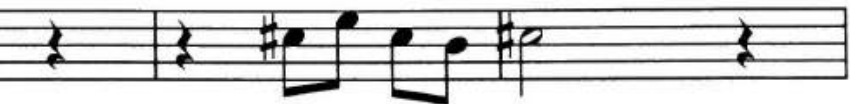


\section{PASILLO}

ALVARO BERMUDEZ

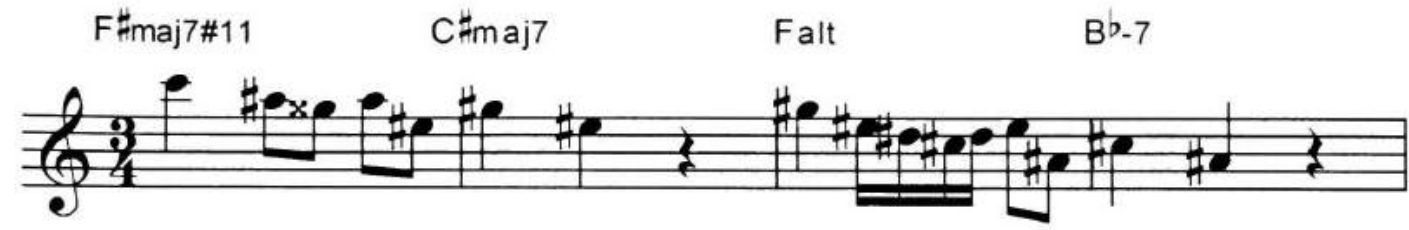
$\mathrm{F}$ maj7\#11
CFmaj7
Falt
$B^{b}-7$

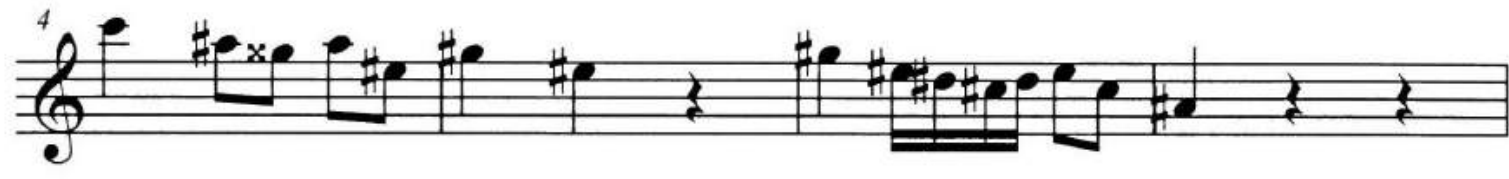
C-9
Eb-maj7
$B^{b / D}$

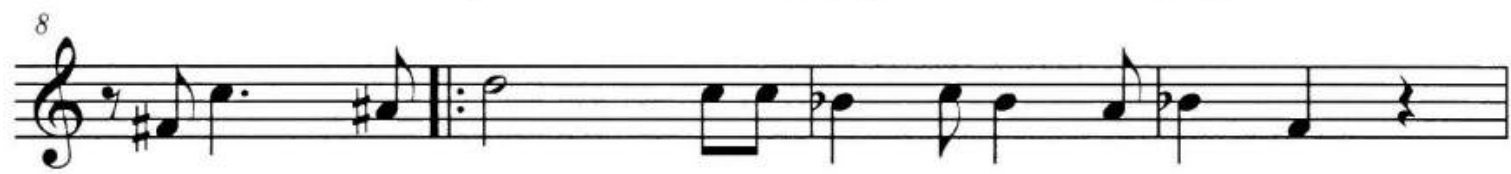
$C \$ 13$
$G \#-9$
CFैalt
Bb-7b5

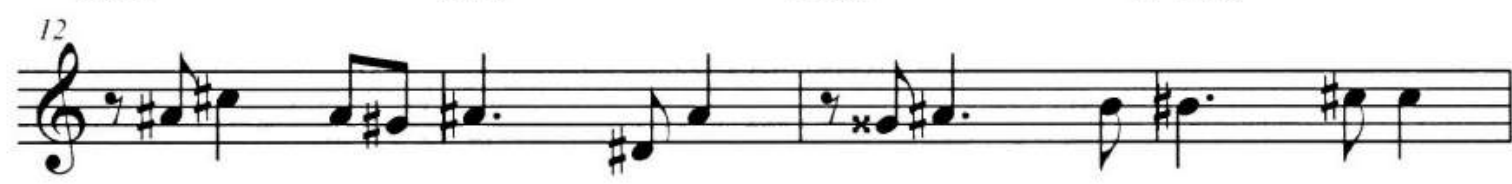

Ebalt

E-7

A9

$F=-7$

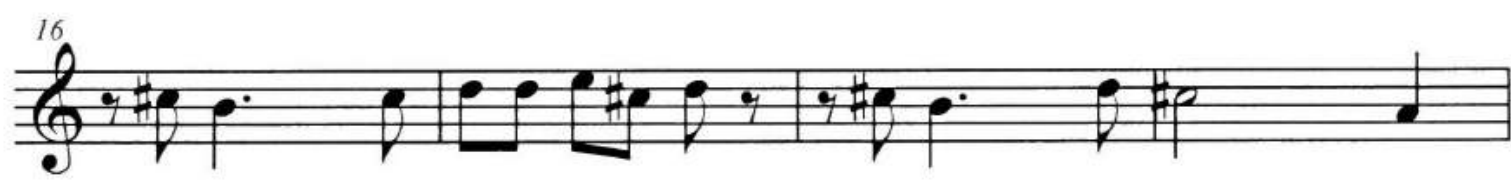
Balt
FËmaj7
Fsus

$B^{b \min 7}$

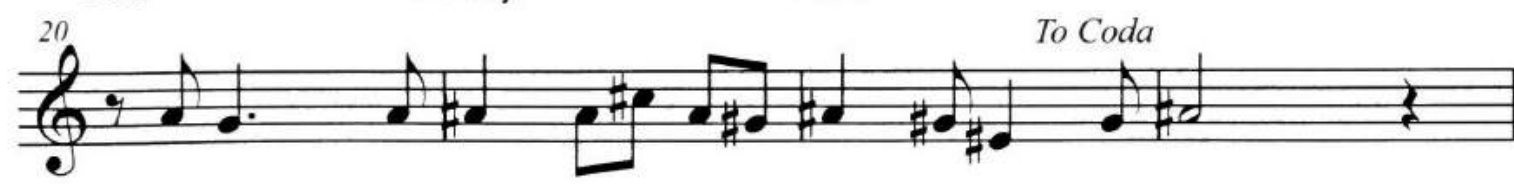



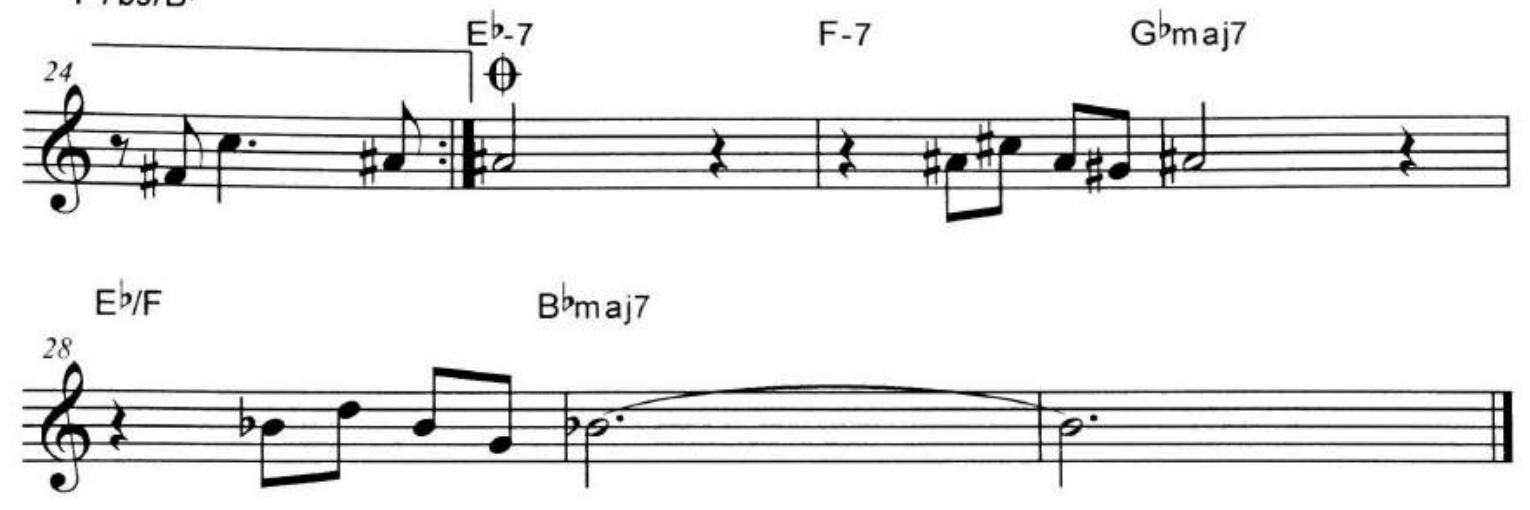


\section{DANZA}

Alvaro Bermudez

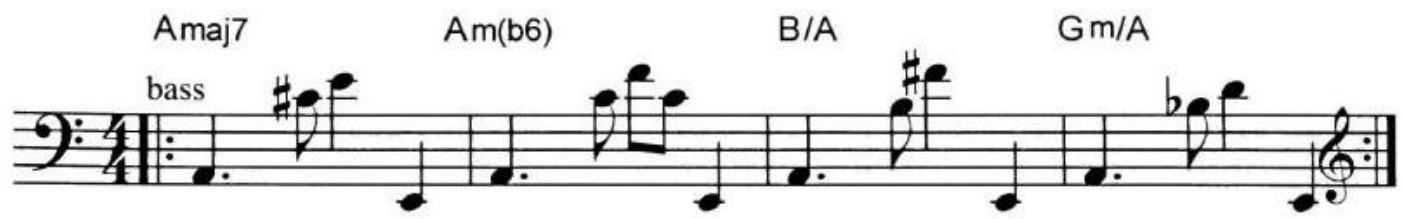
$\oiint_{\text {Amaj7 }}$
$A m(b 6)$
B/A
$\mathrm{Gm} / \mathrm{A}$

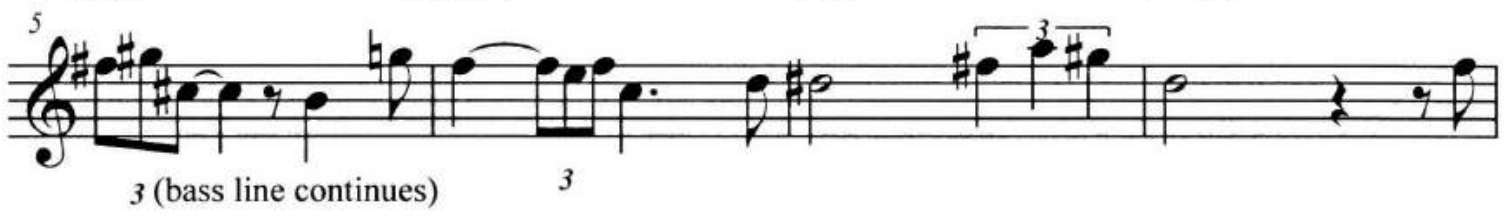
Amaj7
Am(b6)
C\#m9
$B^{b} 6$
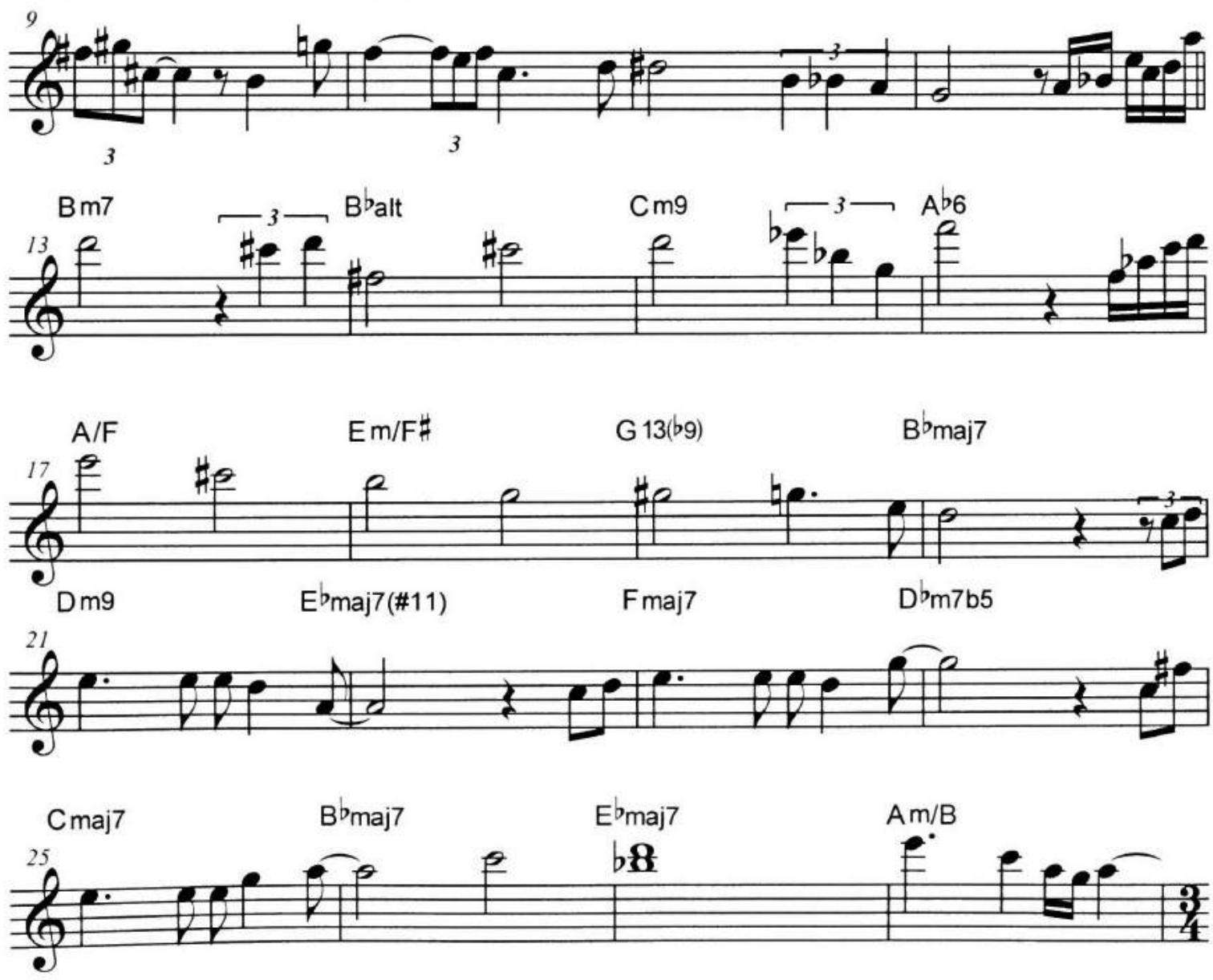
III. Danza

Amaj7

Am(b6)

B/A

$\mathrm{Gm} / \mathrm{A}$

(a) 


\section{DANZA}

Alvaro Bermudez

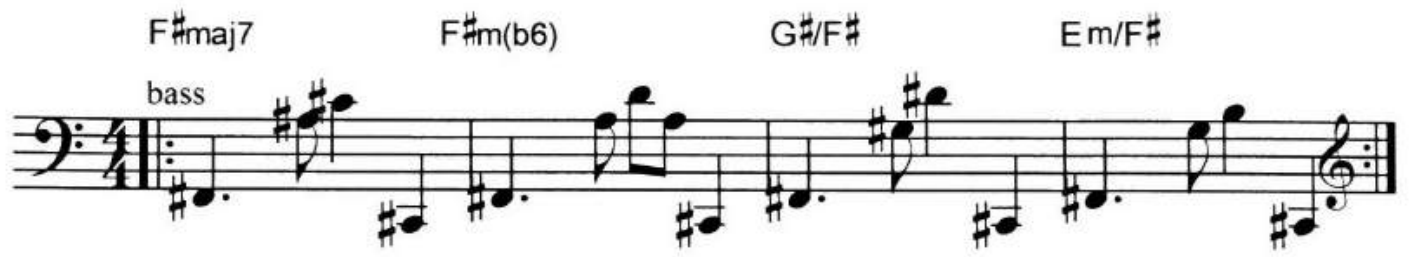

\& $F \not$ maj7 $F \sharp m(b 6) \quad G \# / F \# \quad E m / F \#$
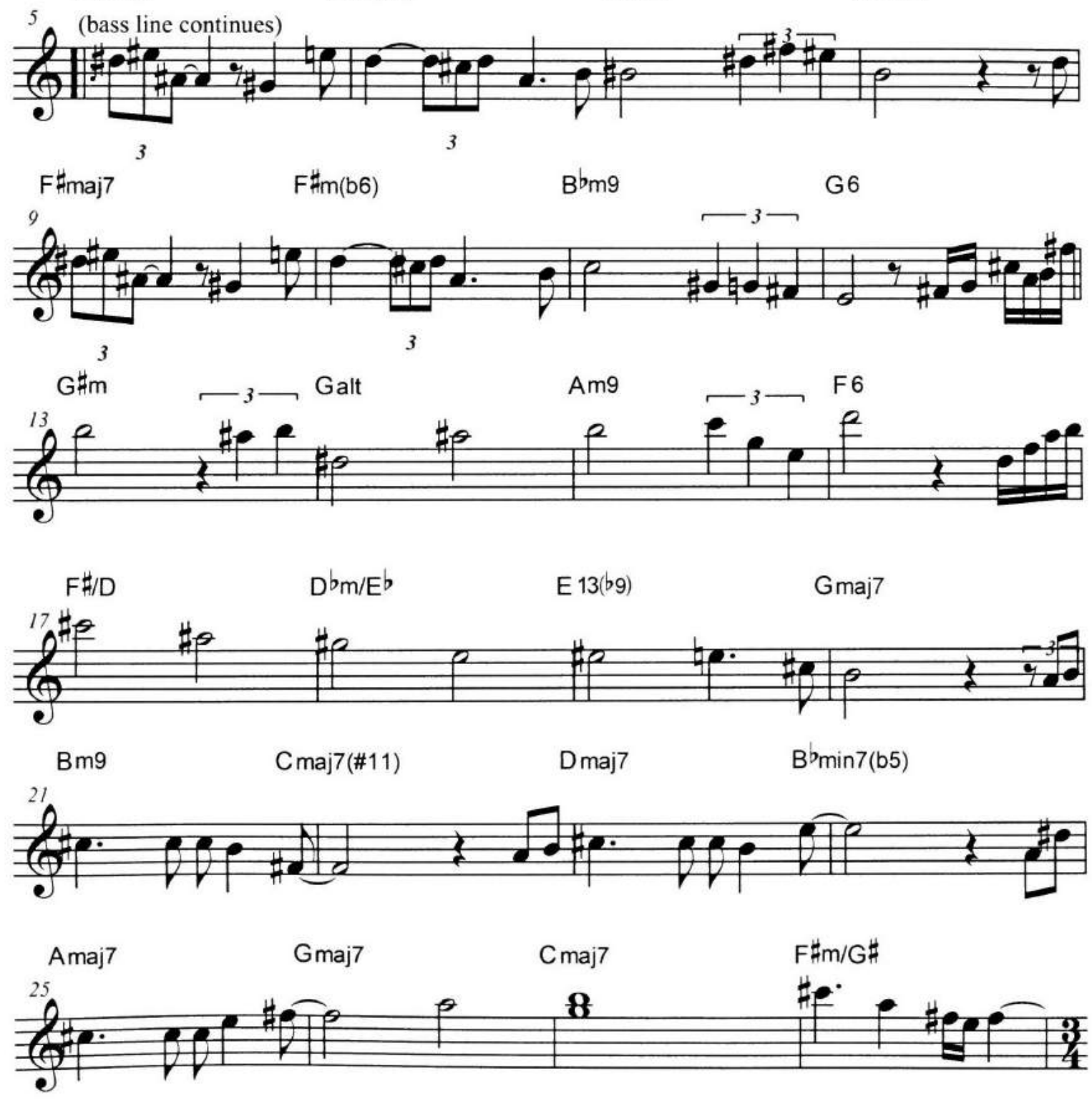
III. Danza

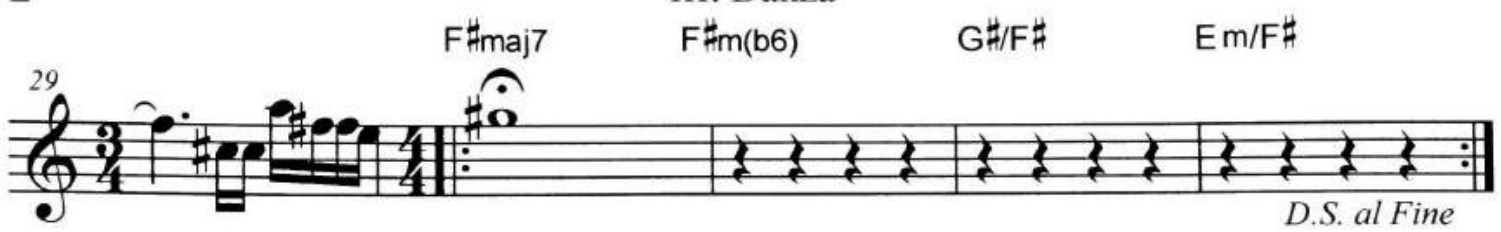


IV. VALS

Alvaro Bermudez
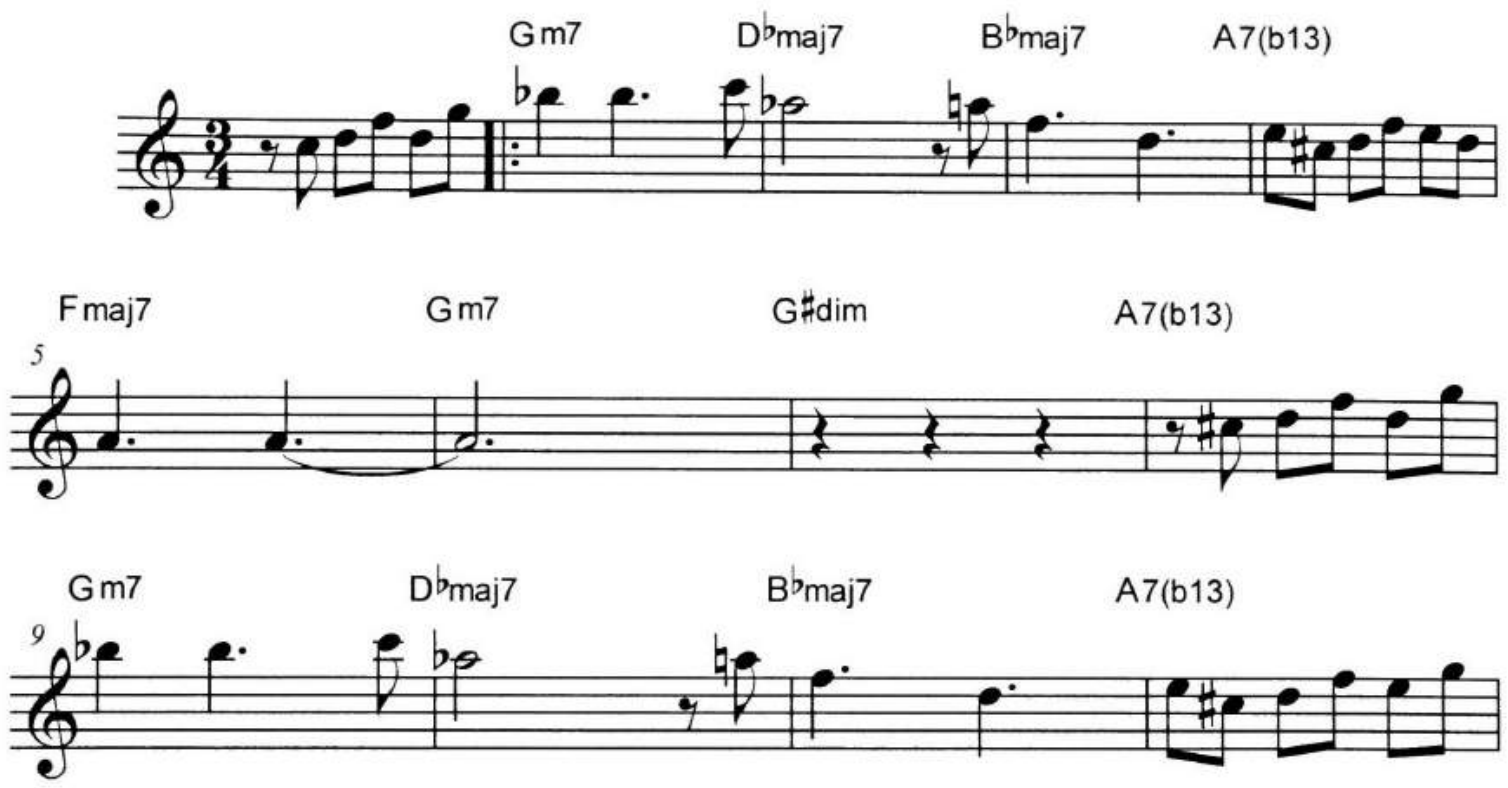

$B \min 7$

E9

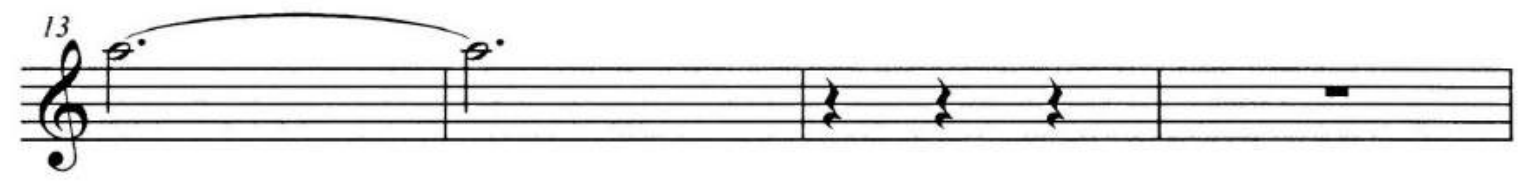

$B \min 7$ (b5)

$B^{\text {Pmaj7 }}$

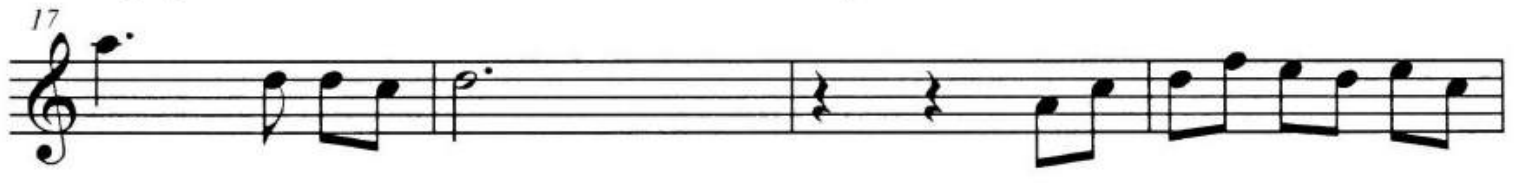

Asus 7

$A b_{\text {maj7(\#11) }}$

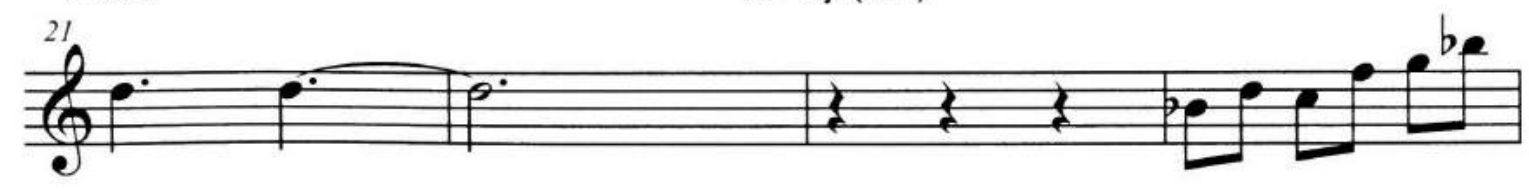



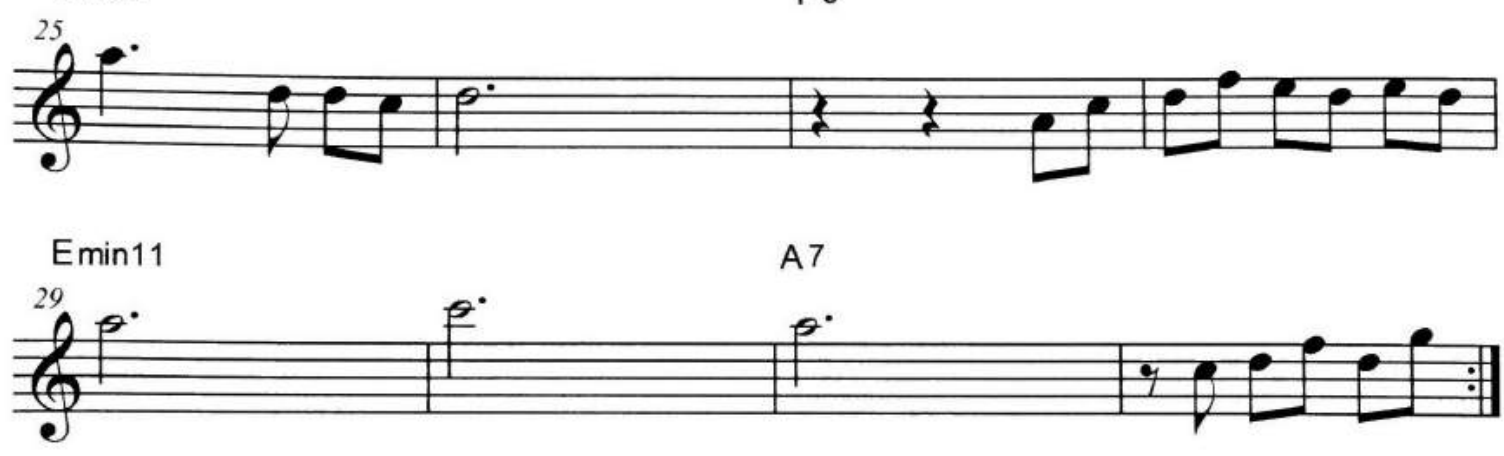

A 7
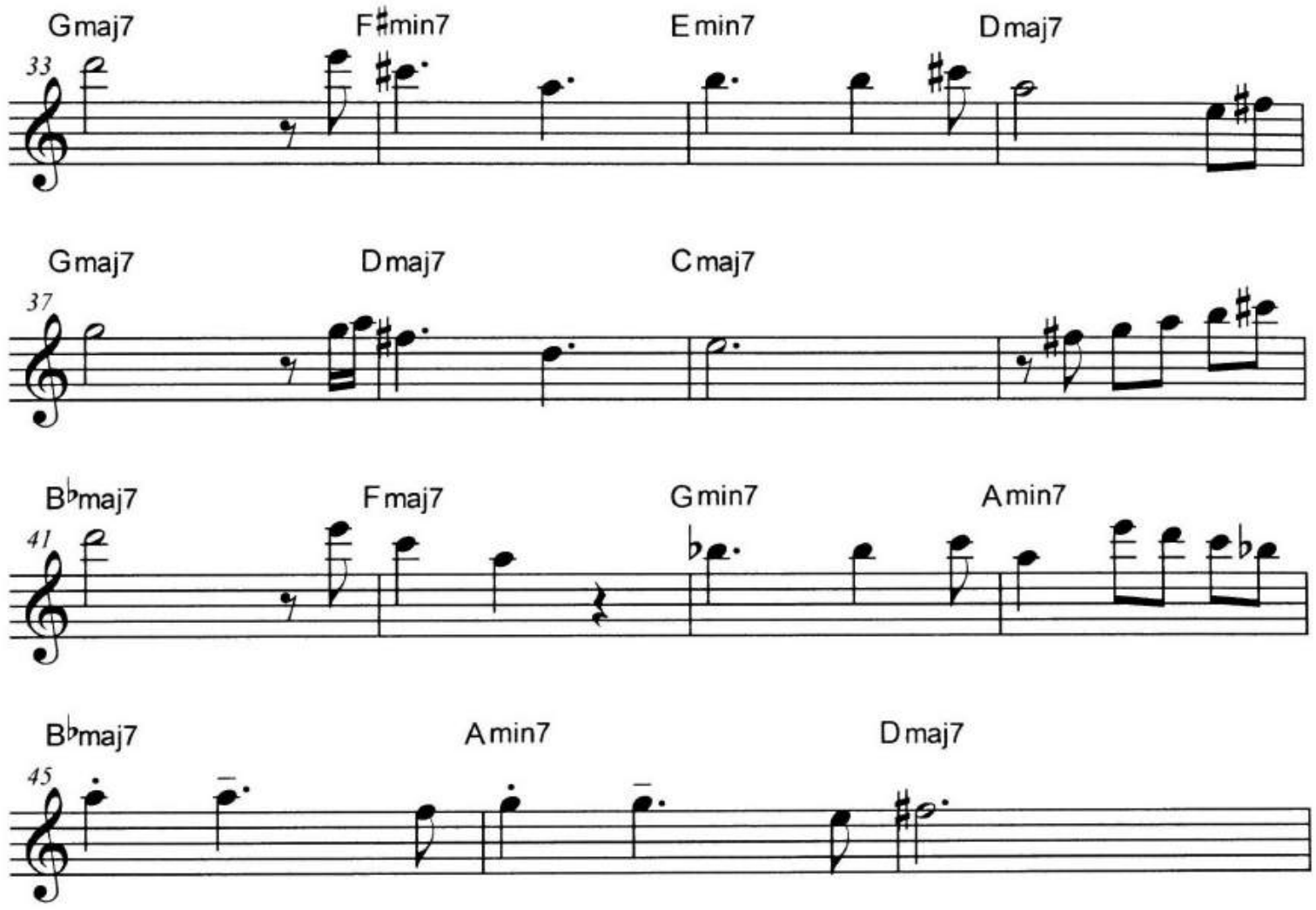

t? 
IV. VALS

\section{Alvaro Bermudez}
Em7
$B^{\text {bmaj7 }}$
Gmaj7
$F \sharp 7$ (b13)
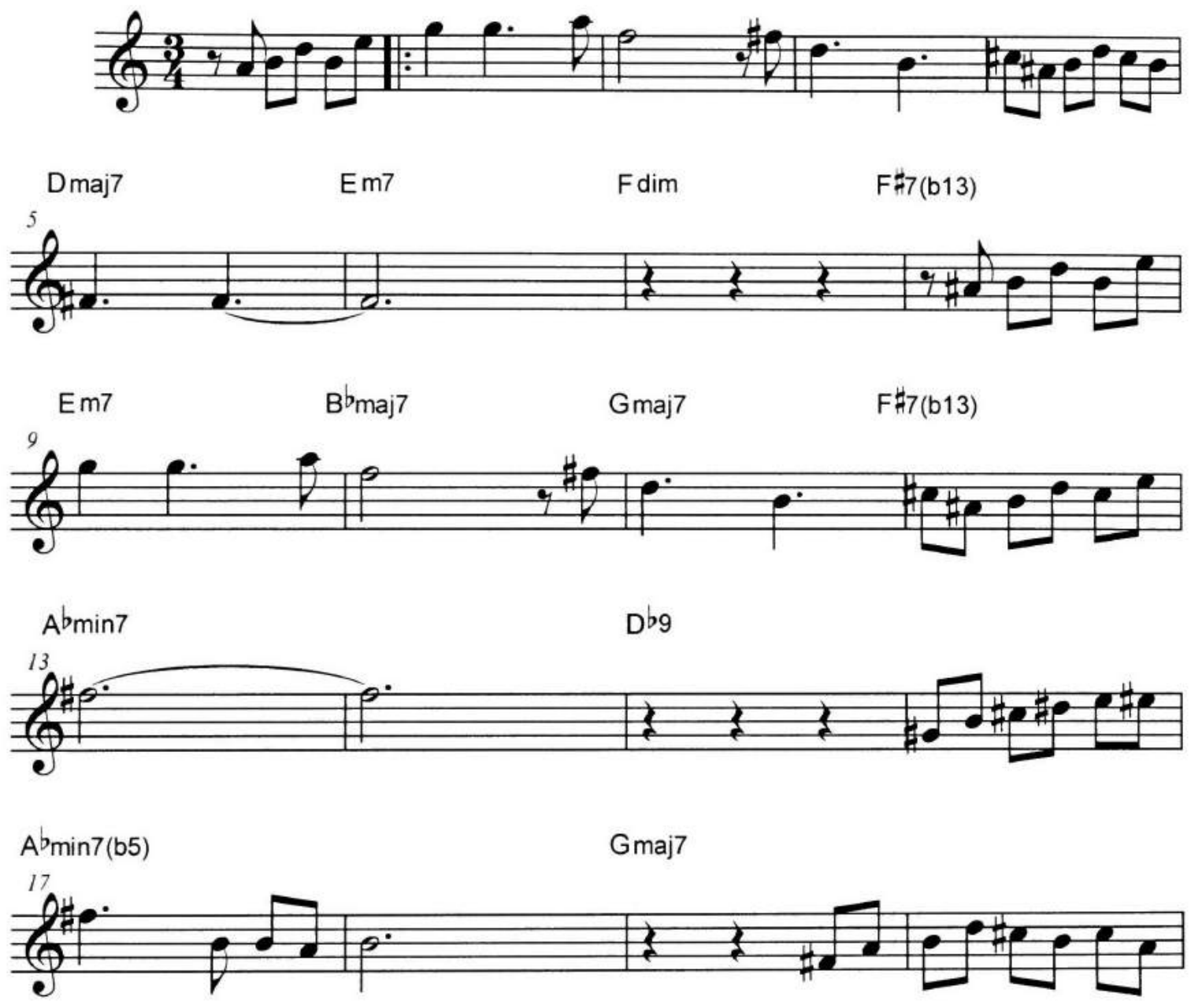

F \#̈sus7

Fmaj7(\#11)

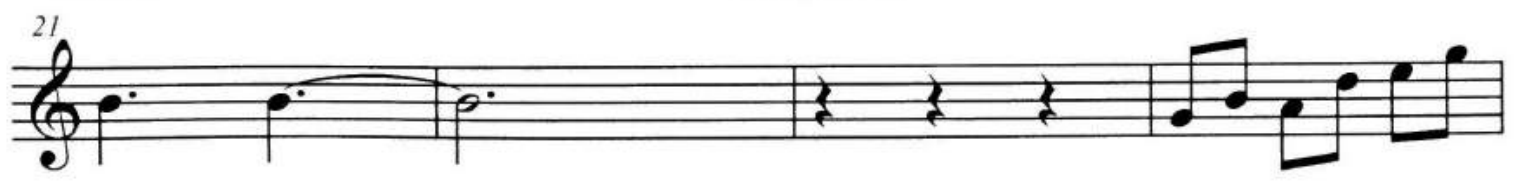



Emin7
D6

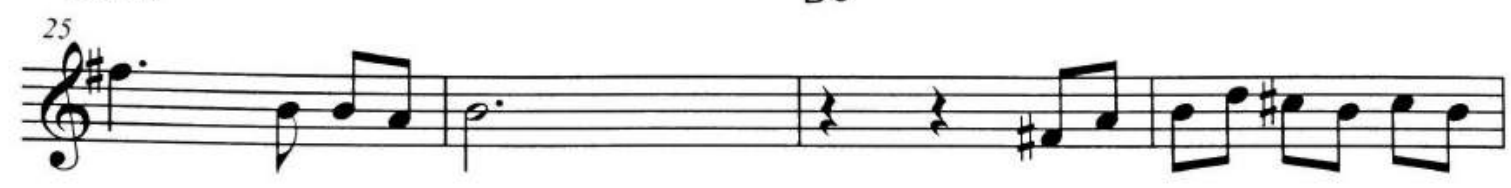
C\#min 7
$\mathrm{F} \# 7$

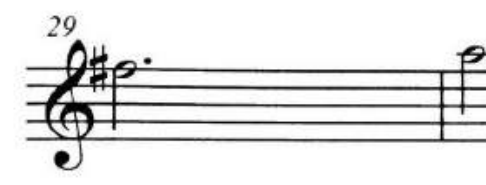

\section{F}
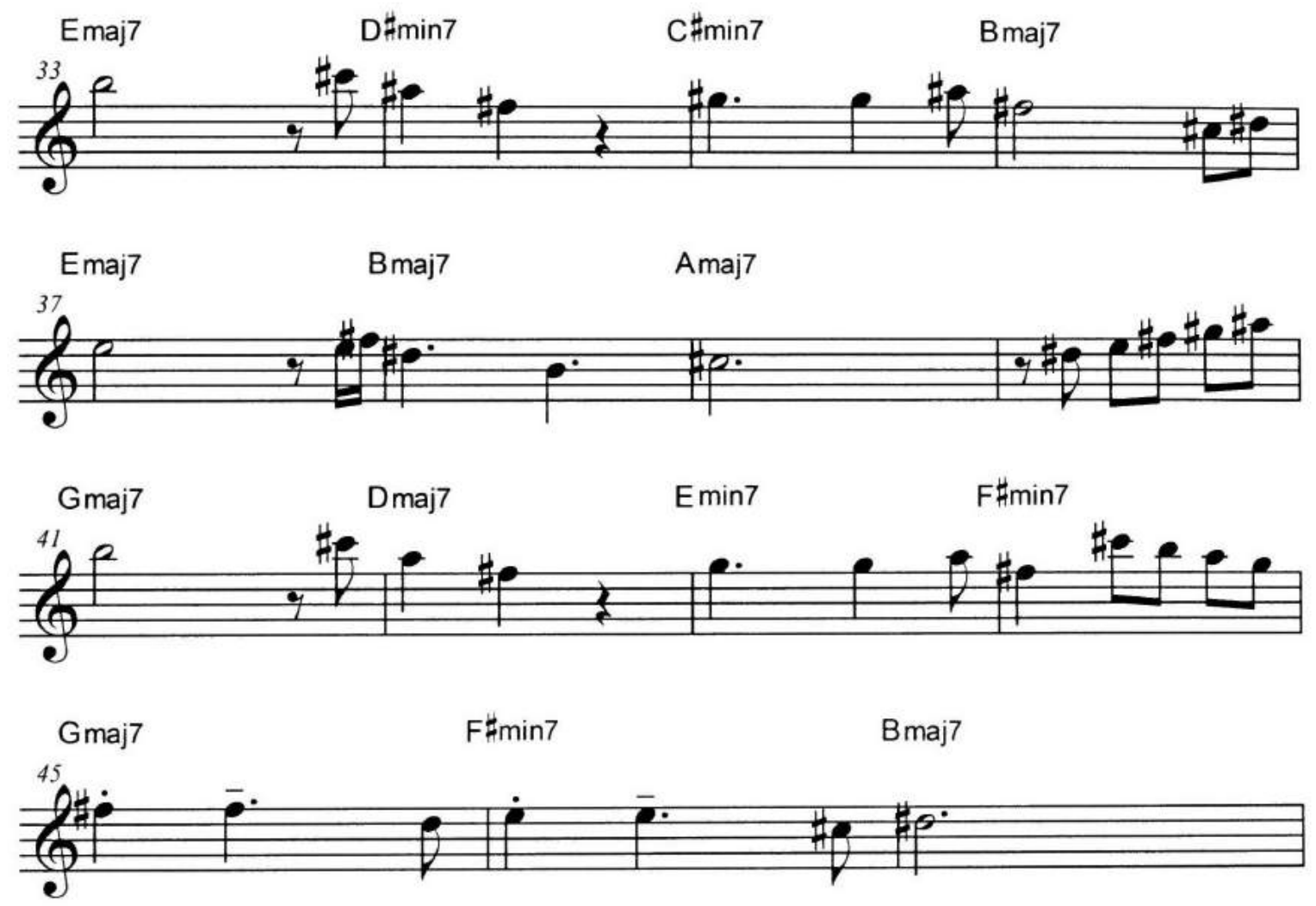

b. 


\section{PASILLO FIESTERO}

Alvaro Bermudez
$\mathrm{F} \min 7$
D'maj7
C7b9

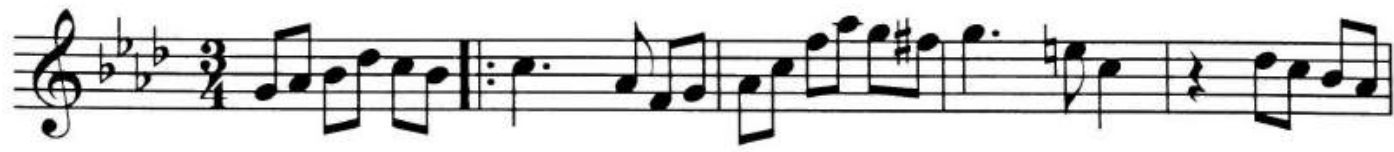

Calt $\mathrm{F} \min 7$
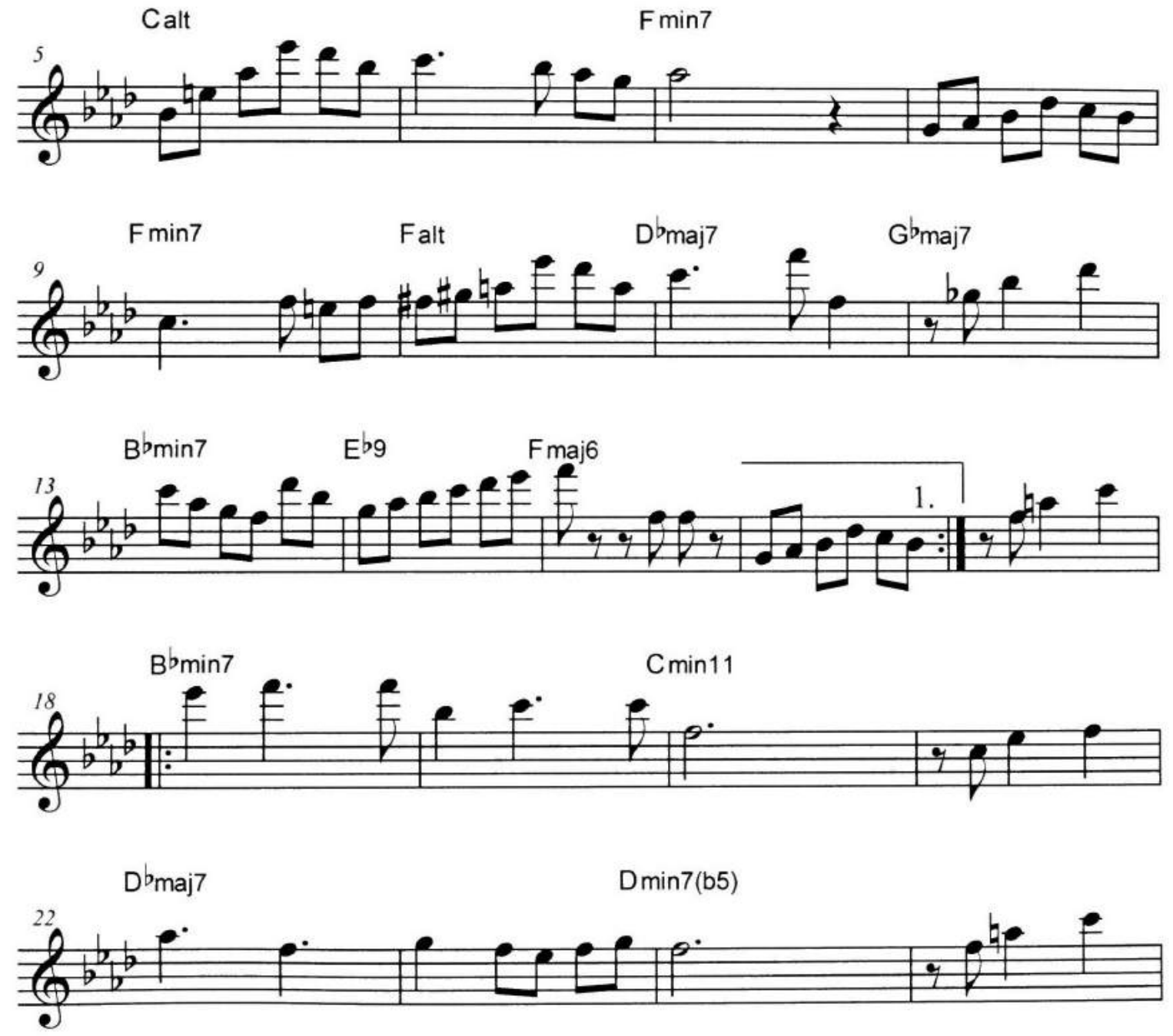

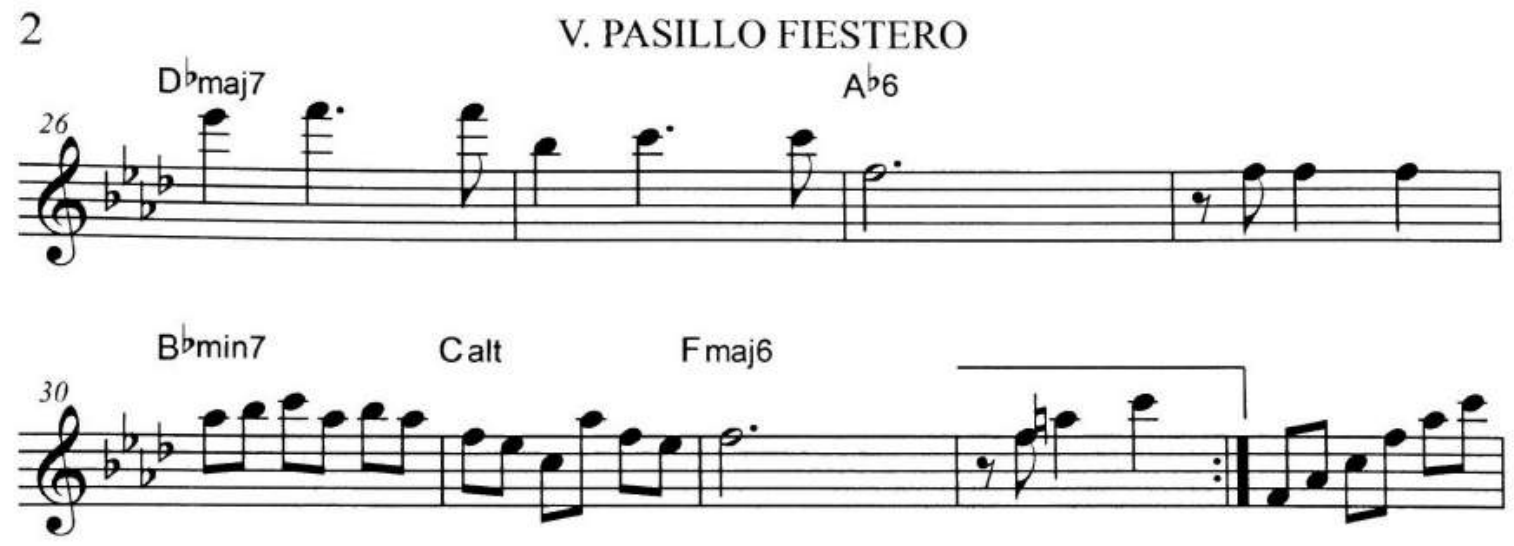

$\mathrm{B}^{\mathrm{b} \min 7(\mathrm{~b} 5)}$

$\mathrm{F} \min 7$

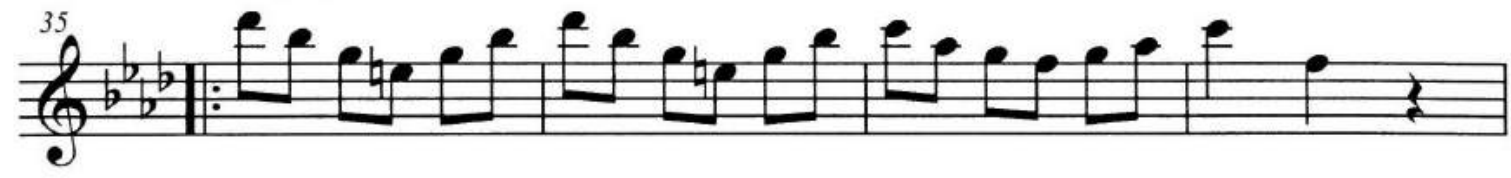

C7b9 Falt
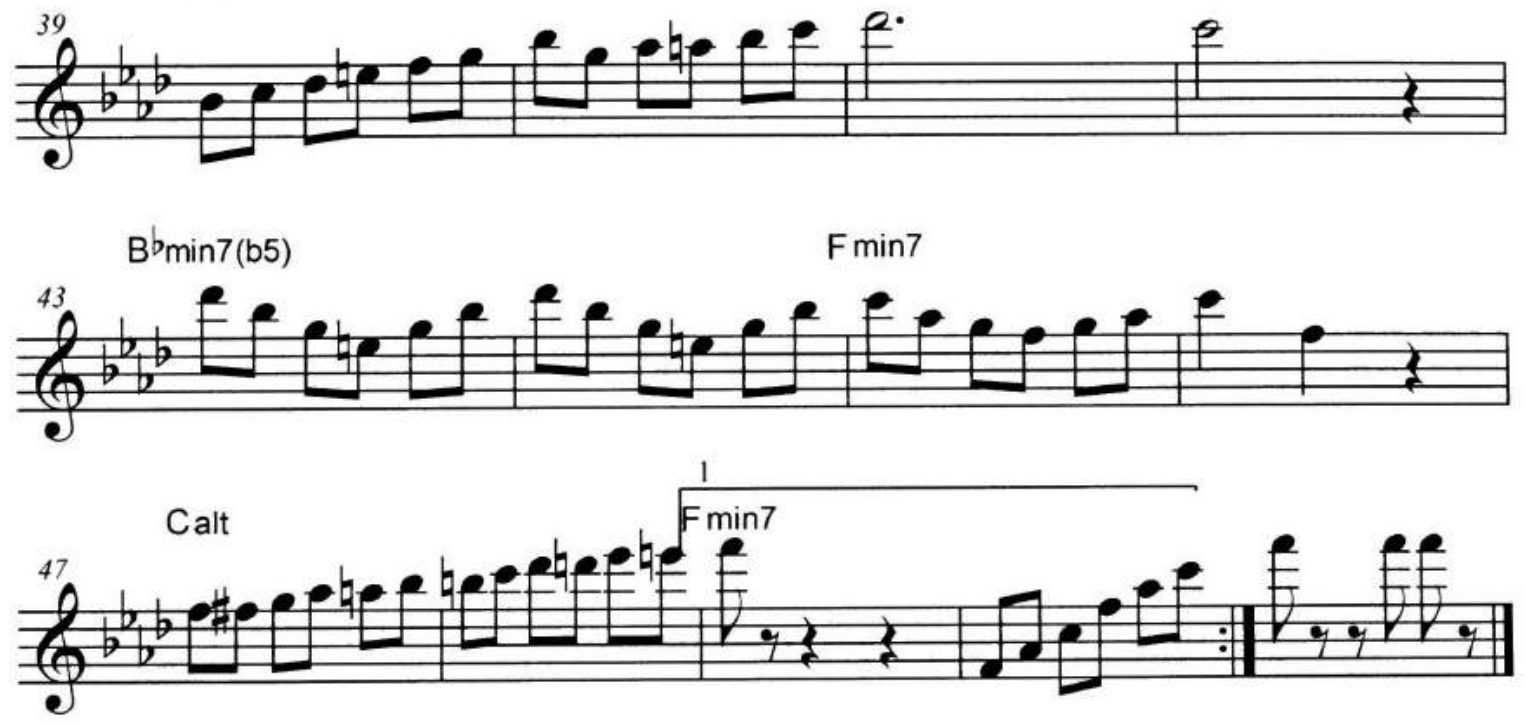


\section{PASILLO FIESTERO}

Alvaro Bermudez

$D \min 7$

$B^{P}$ maj7

A7b9

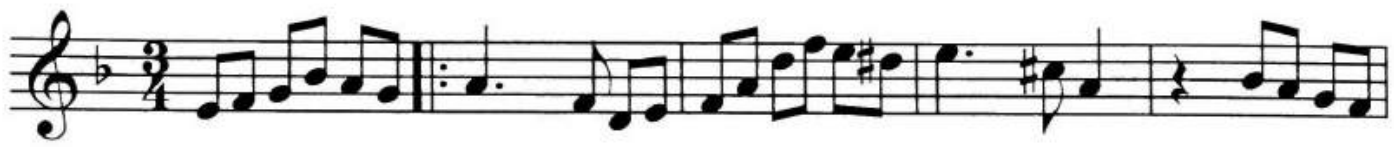

Aalt

$D \min 7$

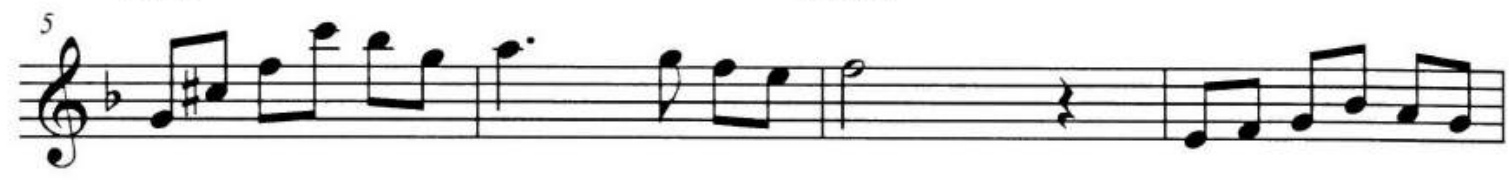

D min7

Dalt

$B^{\text {maj7 }}$

E'maj7

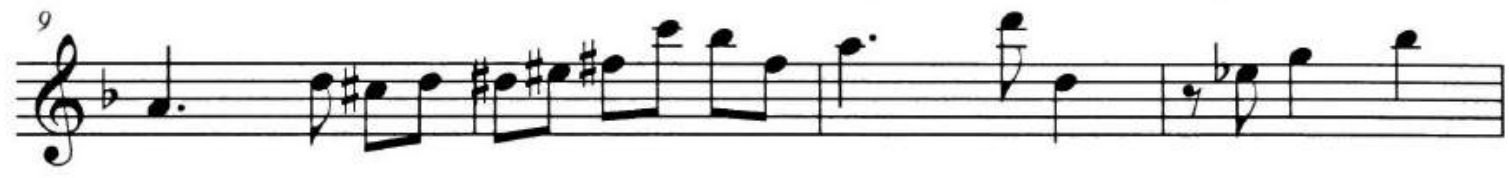

$\mathrm{G} \min 7$

C9 Dmaj6
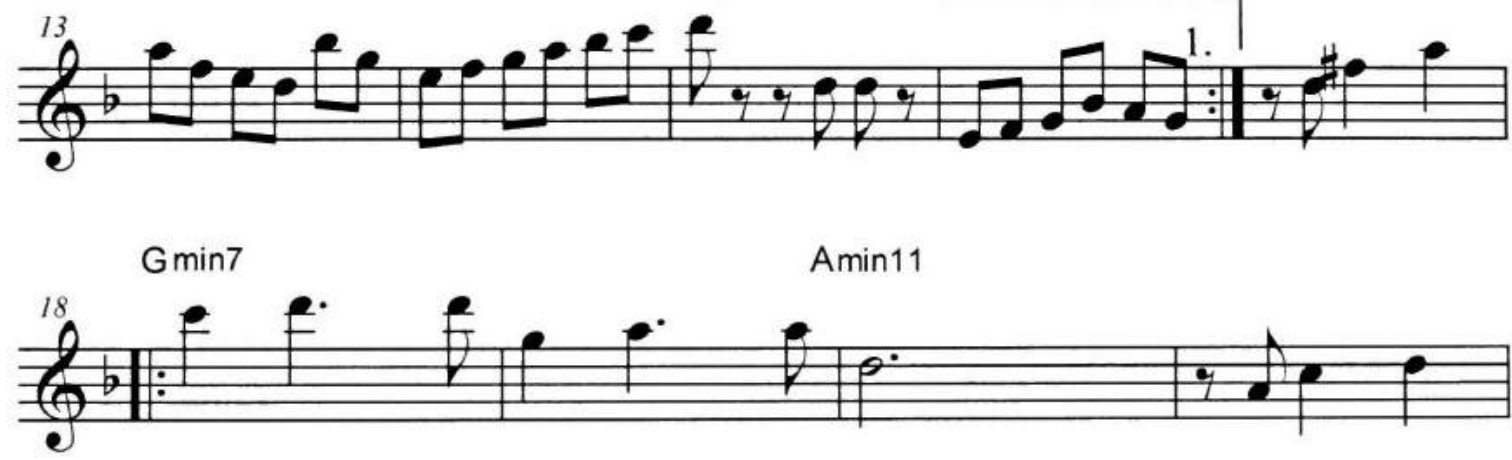

$B^{b_{\text {maj7 }}}$

$B \min 7$ (b5)

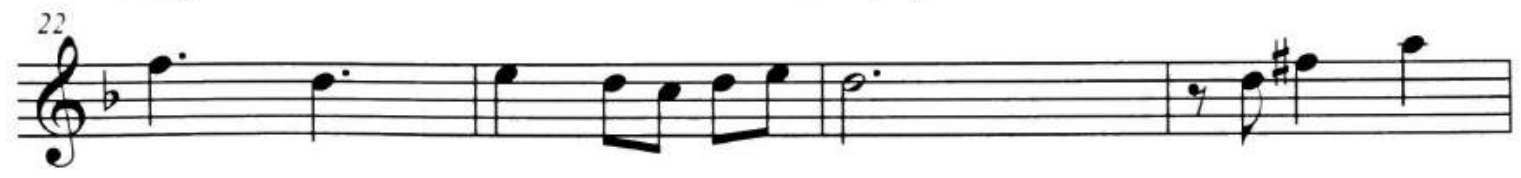



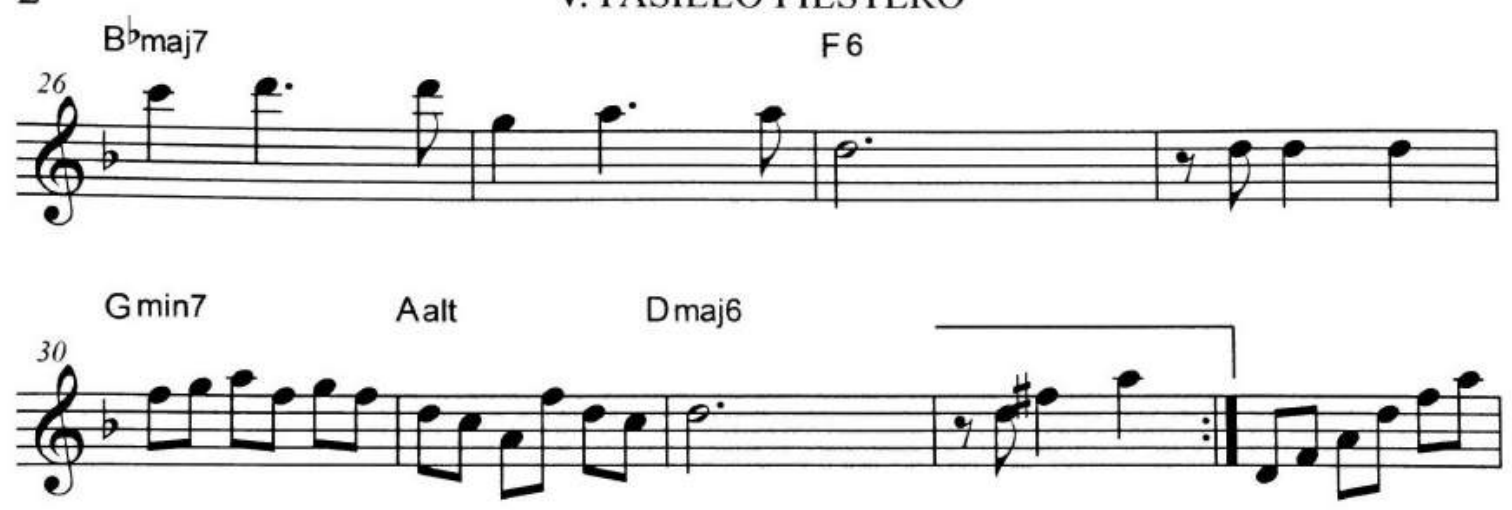

Gmin7(b5)

$D \min 7$

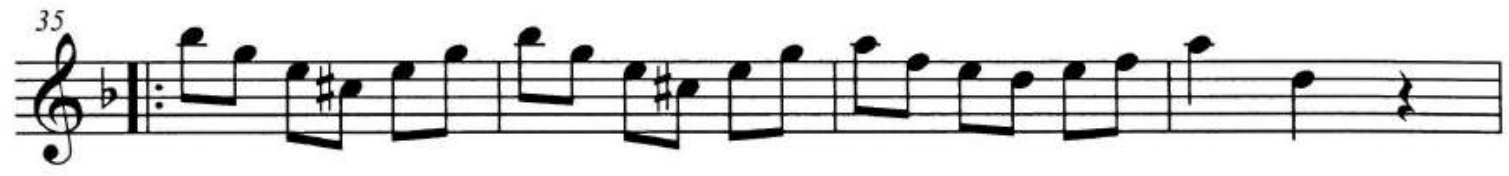

A7b9

Dalt

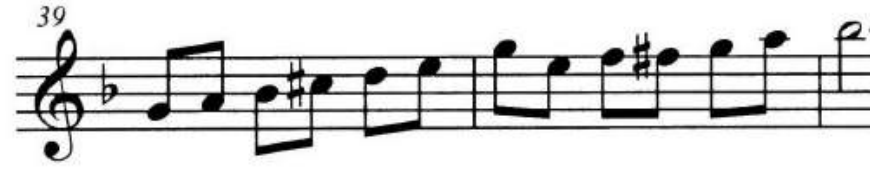

Gmin7(b5)

$D \min 7$
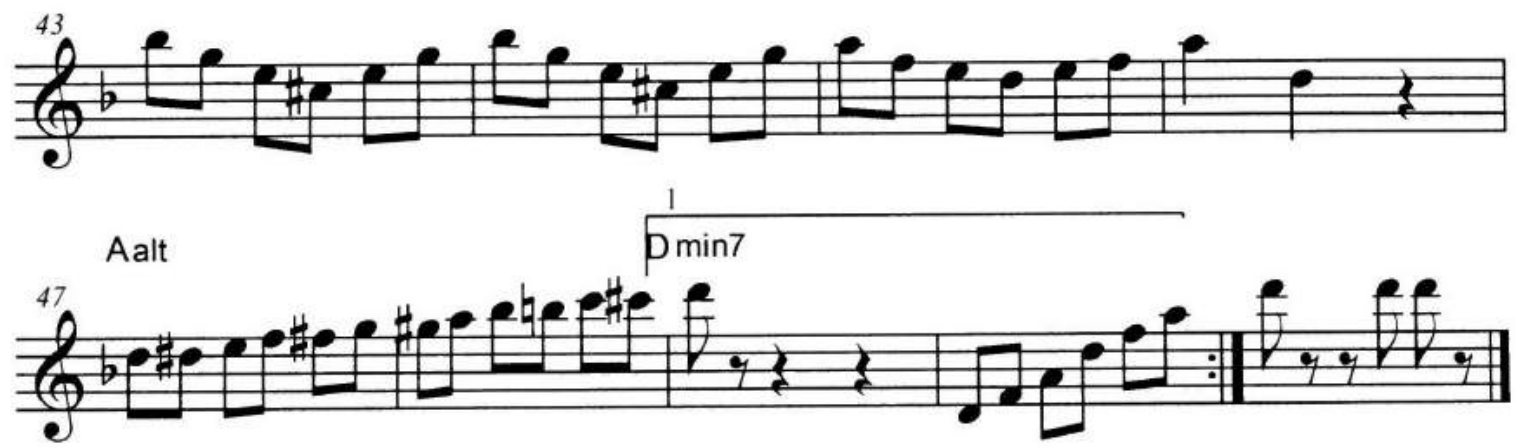


\section{CURRULAO}

(Band builds $\mathrm{G}$ pedal to sax cue)

Alvaro Bermudez
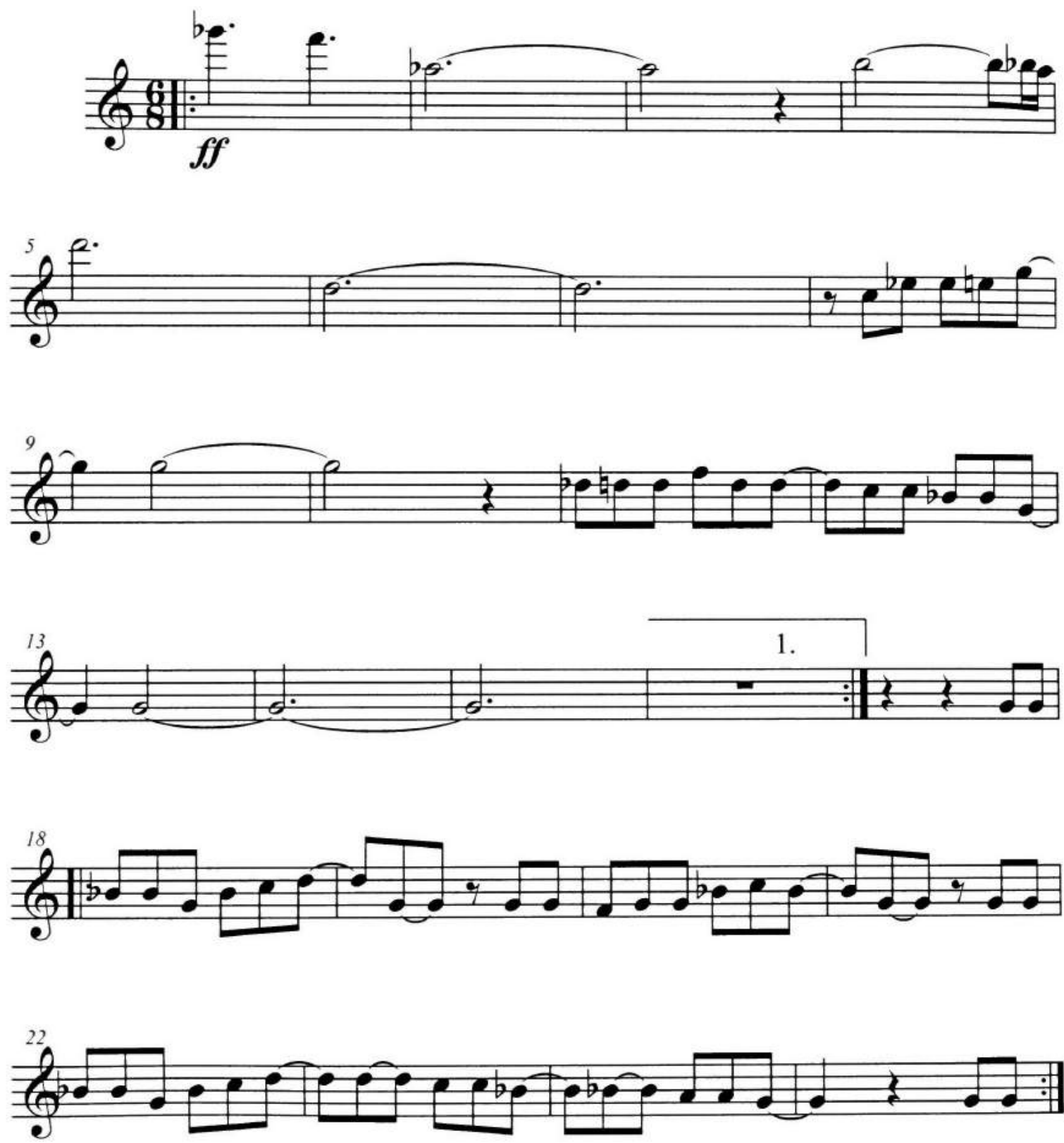

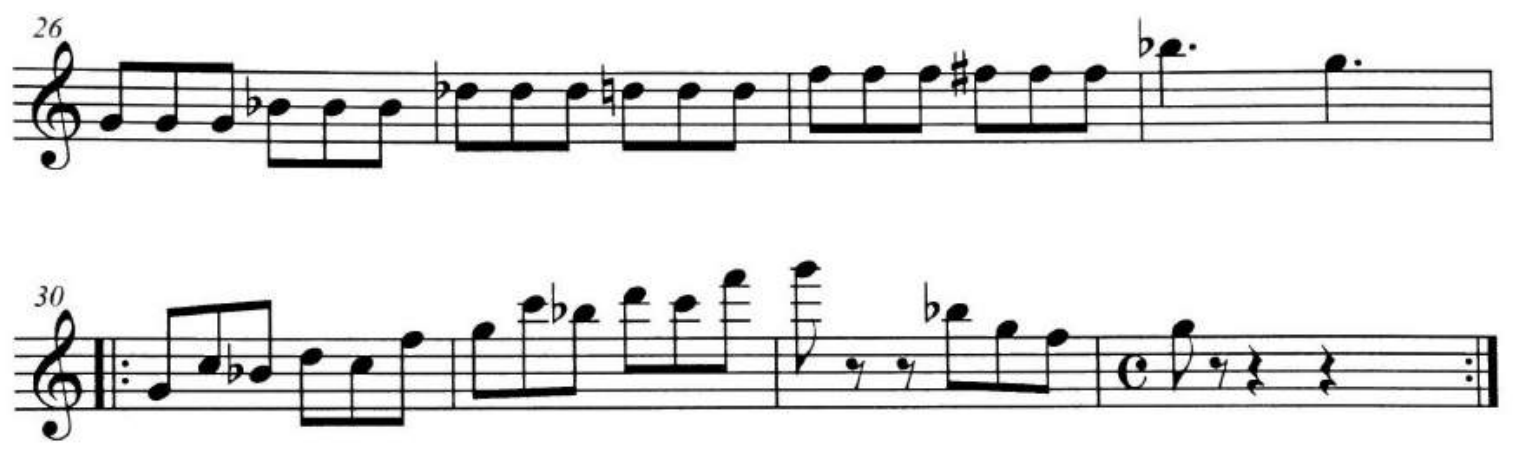

solo on $\mathrm{G}$ minor blues 


\section{CURRULAO}

Alvaro Bermudez
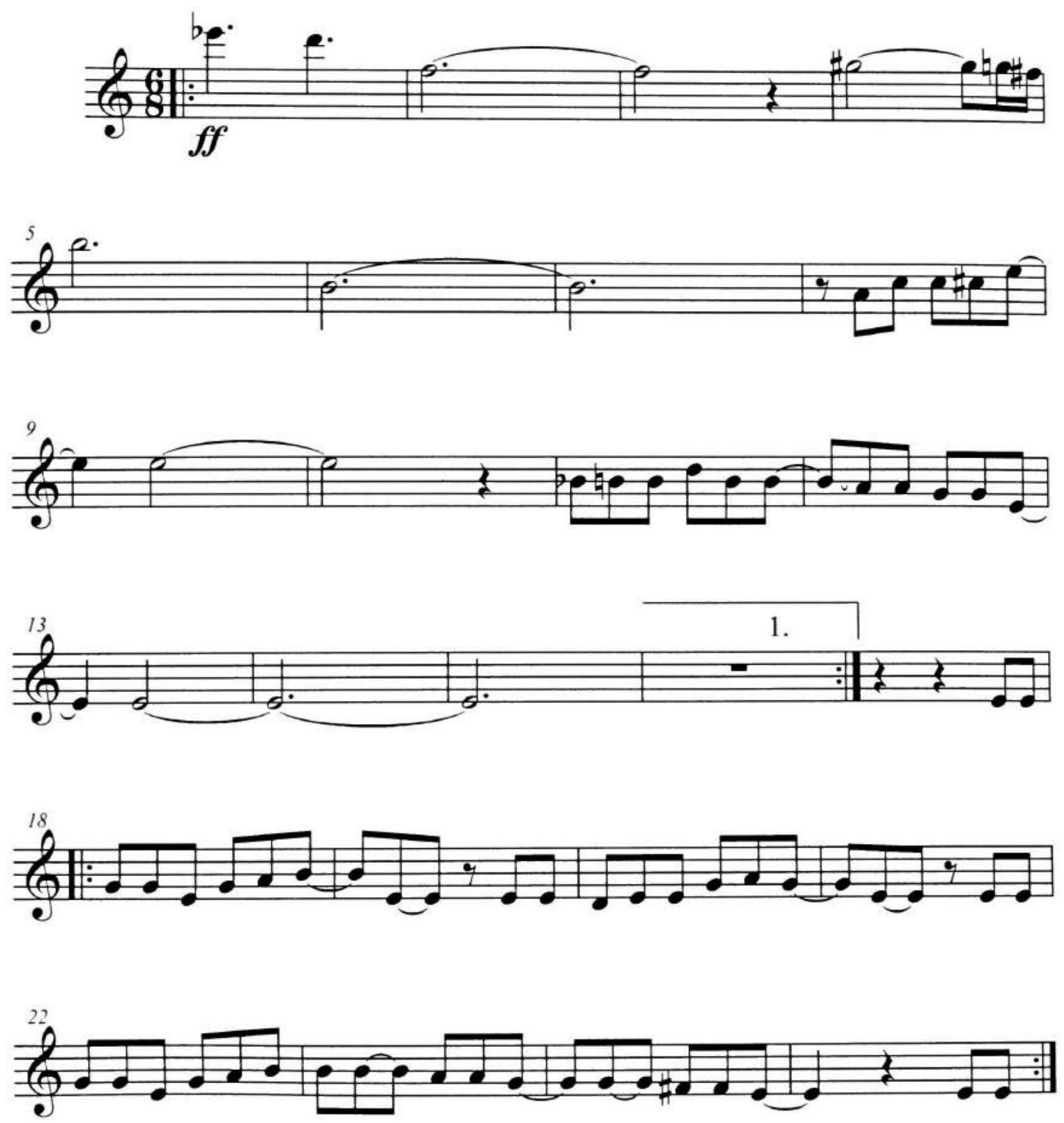

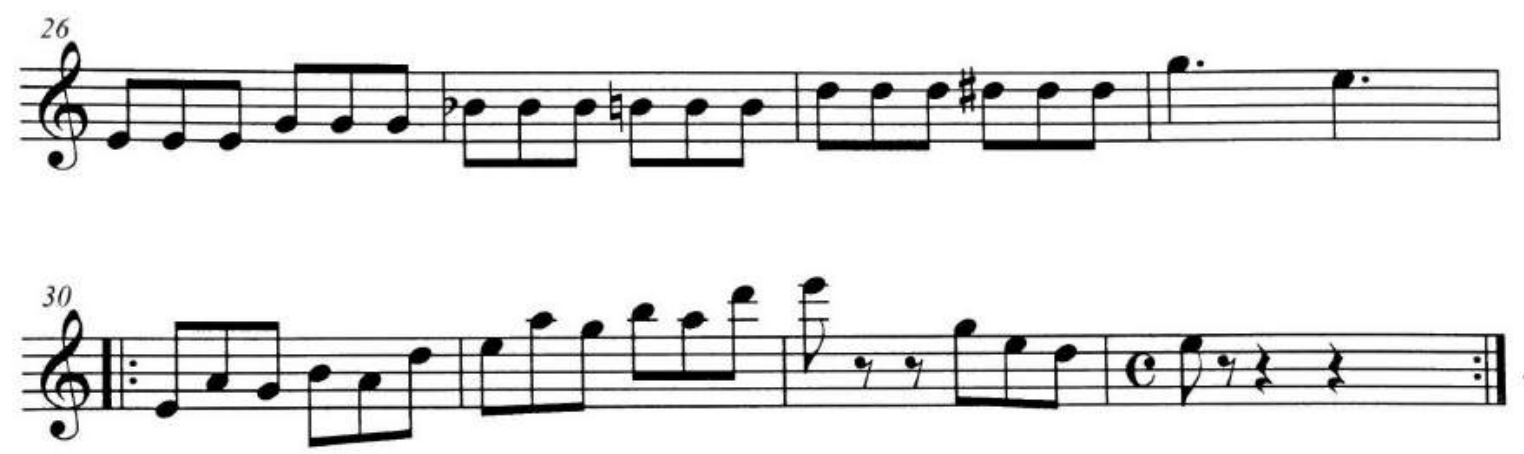

solo on $\mathrm{E}$ minor blues 